\title{
Trapping polar molecules in an ac trap
}

\author{
Hendrick L. Bethlem, ${ }^{1,2, *}$ Jacqueline van Veldhoven, ${ }^{1,3}$ Melanie Schnell, ${ }^{1}$ and Gerard Meijer ${ }^{1}$ \\ ${ }^{1}$ Fritz-Haber-Institut der Max-Planck-Gesellschaft, Faradayweg 4-6, D-14195 Berlin, Germany \\ ${ }^{2}$ Laser Centre Vrije Universiteit, De Boelelaan 1081, NL-1081HV Amsterdam, The Netherlands \\ ${ }^{3}$ FOM-Institute for Plasmaphysics Rijnhuizen, P.O. Box 1207, NL-3430 BE Nieuwegein, The Netherlands
}

(Received 29 September 2006; published 5 December 2006)

\begin{abstract}
Polar molecules in high-field seeking states cannot be trapped in static traps as Maxwell's equations do not allow a maximum of the electric field in free space. It is possible to generate an electric field that has a saddle point by superposing an inhomogeneous electric field to an homogeneous electric field. In such a field, molecules are focused along one direction, while being defocused along the other. By reversing the direction of the inhomogeneous electric field the focusing and defocusing directions are reversed. When the fields are being switched back and forth at the appropriate rate, this leads to a net focusing force in all directions. We describe possible electrode geometries for creating the desired fields and discuss their merits. Trapping of ${ }^{15} \mathrm{ND}_{3}$ ammonia molecules in a cylindrically symmetric ac trap is demonstrated. We present measurements of the spatial distribution of the trapped cloud as a function of the settings of the trap and compare these to both a simple model assuming a linear force and to full three-dimensional simulations of the experiment. With the optimal settings, molecules within a phase-space volume of $270 \mathrm{~mm}^{3}(\mathrm{~m} / \mathrm{s})^{3}$ remain trapped. This corresponds to a trap depth of about $5 \mathrm{mK}$ and a trap volume of about $20 \mathrm{~mm}^{3}$.
\end{abstract}

DOI: 10.1103/PhysRevA.74.063403

PACS number(s): 33.80.Ps, 33.55.Be, 39.10.+j

\section{INTRODUCTION}

Trapping particles has proven a successful strategy for the study of their properties and interactions. Traps allow long interaction times and, therefore, potentially high resolution in spectroscopic and other types of measurements. Not surprisingly, trapped particles are used in various precision tests of fundamental physics theories. Moreover, as the particles are thermally isolated from the outside world, they can be cooled to very low temperatures, making it possible to study cold collisions and create quantum degenerate gases.

Traps for neutral molecules can be formed using static inhomogeneous electric and magnetic fields. In these traps, molecules in states that have a positive energy shift in the applied field-so-called low-field seekers-experience a force toward the center of the trap where the field has a minimum. Magnetostatic trapping has been demonstrated for $\mathrm{CaH}[1]$ and $\mathrm{Cs}_{2}$ molecules [2]. Electrostatic trapping has been demonstrated for $\mathrm{ND}_{3}[3]$ and $\mathrm{OH}[4]$ molecules. Although Maxwell's equations allow for a minimum, they do not allow a maximum of a static magnetic or electric field in free space, required to trap molecules in high-field seeking states. It is possible, however, to create an electric field maximum using optical fields. Optical trapping has been demonstrated for $\mathrm{Cs}_{2}$ molecules [5]. Unfortunately, the trap depth and volume of optical traps are rather limited. Recently, we have demonstrated a considerably deeper and larger trap for high-field seeking molecules using ac electric fields [6]. In this paper we describe this trap in more detail.

Trapping molecules in high-field seeking states is of particular interest for two reasons. (i) The ground state of a system is always lowered by an external perturbation. Therefore, the ground state of any molecule is high-field seeking.

\footnotetext{
*Electronic address: rick@ fhi-berlin.mpg.de
}

In the ground state, trap loss due to inelastic collisions is absent, making it possible to cool these molecules further using evaporative or sympathetic cooling. This is particularly relevant as the dipole-dipole interaction is predicted to lead to large cross sections for inelastic collisions for polar molecules in excited rovibrational states [7]. (ii) Molecules composed out of heavy atoms or many light atoms, such as polycyclic hydrocarbons, have small rotational constants. Consequently all states of these molecules become highfield-seeking in relatively small magnetic or electric fields.

Ac-trapping of polar molecules is closely related to focusing beams of polar molecules using arrays of electrostatic lenses in alternating gradient (AG) configuration. Together with M. R. Tarbutt and E. A. Hinds at Imperial College London, our group has recently published a review on AG focusing [8]. Some of the theory incorporated in that paper is duplicated here.

Our paper is organized as follows. In Sec. II we discuss an extension of Earnshaw's theorem, that shows that molecules in high-field-seeking states cannot be trapped in a static electric field and discuss how one can circumvent this theorem by using ac electric fields. In Sec. III we present three electrode geometries suitable for ac trapping. The optimal shape of the electrodes making up one of these geometries is determined in Sec. IV. In Sec. V we consider the motion of the molecules in an ac trapping field and calculate the depth and volume of an idealized ac trap. In Sec. VI we present an experimental study of the stability of an AC trap. We present measurements of the spatial distribution of trapped ${ }^{15} \mathrm{ND}_{3}$ molecules as a function of the settings of the trap and compare these to both a simple model assuming a linear force and to full three-dimensional (3D) simulations of the experiment. A summary of our main conclusions and a discussion of future prospects are given in Sec. VII. 


\section{EARNSHAW'S THEOREM}

For a force field $\vec{F}(\vec{r})$ to keep a particle in static equilibrium around $\vec{r}=\overrightarrow{0}$, two conditions must be met. The applied force must vanish at $\vec{r}=\overrightarrow{0}$, and, for small displacements, the force field should tend to restore the particle toward $\vec{r}=\overrightarrow{0}$. To achieve the latter it is necessary that the divergence of the force be negative, $\vec{\nabla} \cdot \vec{F}<0$ in a volume around $\vec{r}=\overrightarrow{0}$.

The force on a charged particle placed in an electric field is given by $\vec{F}=q \vec{E}$. Since $\vec{\nabla} \cdot \vec{E}=0$ (or $\nabla^{2} V=0$ ), a charged particle cannot be held in stable equilibrium by electrostatic forces alone. This is known as Earnshaw's theorem [9]. The application of Earnshaw's theorem to polar molecules in inhomogeneous electric fields was first discussed by Auerbach, Bromberg, and Wharton [10] in a design study of a neutral particle accelerator. The implications of Earnshaw's theorem for trapping neutral atoms and molecules in electromagnetic fields were discussed by Wing [11] and by Ketterle and Pritchard [12].

The force acting on a polar molecule in an inhomogeneous electric field $\vec{E}(\vec{r})$ is given by

$$
\vec{F}(\vec{r})=-\vec{\nabla} W(E),
$$

with $W(E)$ the Stark energy of a polar molecule in an electric field of magnitude $E=|\vec{E}|$. In most common cases the Stark shift of a molecule is either a linear or quadratic function of the applied field. For molecules that experience a linear Stark shift in the applied field, $W=-\mu_{\mathrm{eff}} E$, it can be shown that [10]

$$
\begin{aligned}
\vec{\nabla} \cdot \vec{F}= & \frac{\mu_{\mathrm{eff}}}{E^{3}} \sum_{i, j, k=1}^{3}\left[\left(\frac{\partial \Phi}{\partial x_{k}}\right)^{2}\left(\frac{\partial^{2} \Phi}{\partial x_{i} \partial x_{j}}\right)^{2}-\left(\frac{\partial \Phi}{\partial x_{i}}\right)\left(\frac{\partial \Phi}{\partial x_{k}}\right)\right. \\
& \left.\times\left(\frac{\partial^{2} \Phi}{\partial x_{i} \partial x_{j}}\right)\left(\frac{\partial^{2} \Phi}{\partial x_{k} \partial x_{j}}\right)\right],
\end{aligned}
$$

where $\Phi$ is the electrostatic potential and $\mu_{\text {eff }}$ is an effective dipole moment which depends on the particular molecular state. Using Schwarz's inequality, it can be seen that the sum is always larger than, or equal to, zero. Therefore, for molecules having a linear Stark shift the sign of $\vec{\nabla} \cdot \vec{F}$ is determined solely by the sign of $\mu_{\text {eff }}$.

Similarly, for molecules that experience a quadratic Stark shift in the applied field, $W=-1 / 2 \alpha_{\text {eff }} E^{2}$, it can be shown that $[10]$

$$
\vec{\nabla} \cdot \vec{F}=\alpha_{\text {eff }} \sum_{i, j=1}^{3}\left(\frac{\partial^{2} \Phi}{\partial x_{i} \partial x_{j}}\right)^{2},
$$

where $\alpha_{\text {eff }}$ is the effective polarizability which depends on the particular molecular state. Again, it is seen that the sign of $\vec{\nabla} \cdot \vec{F}$ is only determined by the sign of $\alpha_{\text {eff }}$.

For molecules in low-field seeking states, i.e., for molecules that have a negative $\mu_{\text {eff }}$ or $\alpha_{\text {eff }}$, it is seen from Eq. (2) and Eq. (3) that $\vec{\nabla} \cdot \vec{F} \leq 0$ and trapping is straightforward. This is equivalent to saying that it is possible to create an electric field minimum. For molecules in high-field seeking states, i.e., for molecules that have a positive $\mu_{\mathrm{eff}}$ or $\alpha_{\mathrm{eff}}$ it is seen from Eq. (2) and Eq. (3) that $\vec{\nabla} \cdot \vec{F} \geq 0$ and trapping is more problematic. This is equivalent to saying that it is not possible to create an electric field maximum. Trapping molecules in high-field seeking states is nevertheless possible using (i) Circular motion. In a storage ring the curvature of the trajectory adds a centrifugal force which, in an appropriately shaped electric field, stabilizes the motion of the molecules, similar to "weak focusing" storage rings for ions [13]. For example, the electric field in a capacitor formed by two coaxial cylinders scales as $1 / r$, with $r$ the distance from the axis. Molecules that have a linear Stark shift in the applied field will experience a force that is proportional to $1 / r^{2}$. These molecules therefore move in stable Kepler-type orbits around the central electrode [14-16]. By shaping the outer cylinder appropriately, molecules are also trapped along the direction of the central electrode $[17,18]$. (ii) Time varying electric fields. Although it is not possible to create a field for which $\vec{\nabla} \cdot \vec{F}<0$ at any given position, it is possible to create a field for which $\vec{\nabla} \cdot \vec{F}$ is equal to zero at a certain position, irrespective of (the sign of) the Stark shift. As a consequence, at this position the focusing force along one direction is equal to the defocusing forces along the other directions, i.e., the electric field strength has a saddle point here. In some cases it is possible to reverse the focusing and defocusing directions by changing the voltages applied to the electrodes. If we switch the voltages between these two configurations, molecules will be alternately focused and defocused. As molecules tend to be farther away from the saddle point along the focusing direction and closer to the saddle point along the defocusing direction, this leads to a net focusing. This principle is used in ion traps [19] and alternate gradient synchrotrons [20].

From this discussion, we see that in order to circumvent Earnshaw's theorem we need to introduce some form of motion or time variation [11]. This can be either motion of the molecule-in case of trapping molecules in a storage ring - or a time variation of the applied electric field-in case of an ac trap.

\section{ELECTRODE GEOMETRY}

In Fig. 1 three electrode geometries are depicted that are suitable for ac trapping of polar molecules. These geometries have in common that the symmetry of the electrostatic potential is such that $\vec{\nabla} \cdot \vec{F}=0$ at the center, and that the direction of $\vec{F}$ can be reversed by changing the voltages applied to the electrodes [47].

\section{A. Linear ac trap}

Consider the geometry shown in Fig. 1(a). The four identical electrodes are positioned on the corners of a square. A positive voltage is applied to the upper electrode, while an equal negative voltage is applied to the lower electrode. The other two electrodes are at ground potential [48]. Molecules in high-field-seeking states are focused toward the center along the $x$ axis while they are defocused along the $y$ axis. We will first assume the electrodes to be of infinite length, in which case $\partial \Phi / \partial z=0$ and $\partial^{2} \Phi / \partial z^{2}=0$. The electrostatic po- 


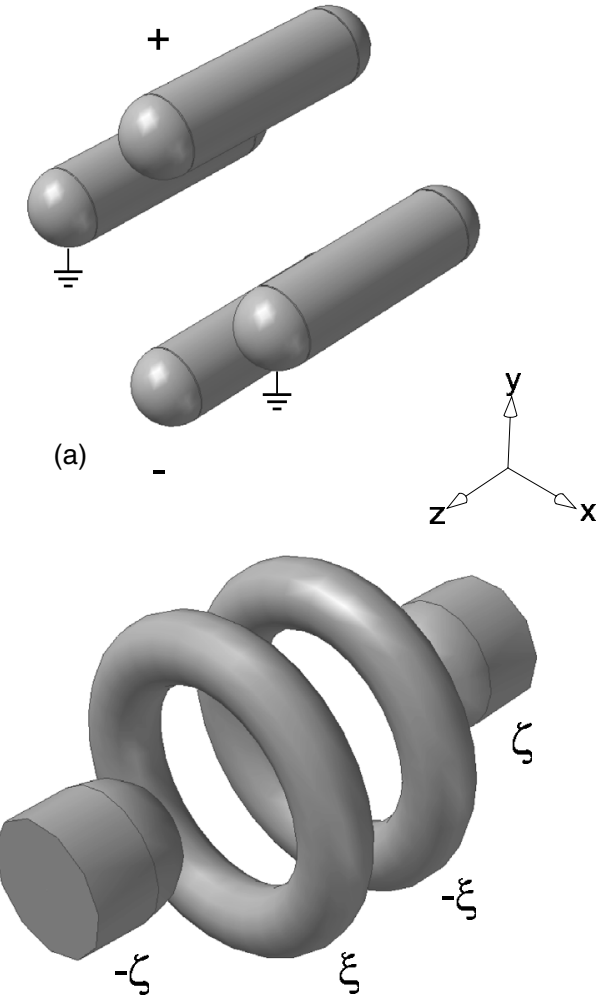

(b)

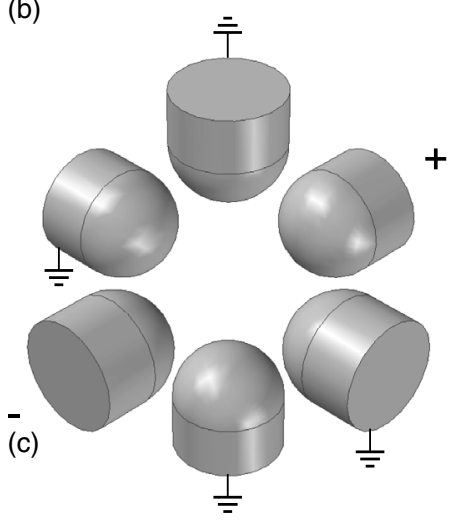

FIG. 1. Three possible electrode geometries that can be used for ac trapping of polar molecules. (a) Linear ac trap. (b) Cylindrical ac trap. (c) Three-phase ac trap.

tential is symmetric under reflection in the $y-z$ plane and therefore $\partial \Phi / \partial x=0$ everywhere on this plane. The electrostatic potential is antisymmetric under reflection in the $x-z$ plane, and therefore $\partial^{2} \Phi / \partial y^{2}=0$ everywhere on this plane. At the intersection of the two planes, the $z$ axis, both $\partial^{2} \Phi / \partial y^{2}=0$ and $\partial^{2} \Phi / \partial z^{2}=0$. As the electrostatic potential obeys Laplace's equation, $\nabla^{2} \Phi=0$, it follows that $\partial^{2} \Phi / \partial x^{2}$ $=0$ at the $z$ axis. Using these relations we find from Eq. (2) and Eq. (3) that for molecules experiencing a linear or quadratic Stark shift $\vec{\nabla} \cdot \vec{F}=0$ at the $z$ axis. The force constants in the two transverse directions, $k_{x}=-\partial F / \partial x$ and $k_{y}=-\partial F / \partial y$, are therefore equal and opposite, $k_{x}=-k_{y}$. The focus and defocus direction can be reversed by switching the voltage off on the upper and lower electrodes while applying a voltage difference to the two electrodes that were initially at ground potential. By alternating between these two configurations at the appropriate frequency, this geometry acts as $2 \mathrm{D}$ guide for polar molecules in both high-field and low-field seeking states. This guide was first proposed by Auerbach, Bromberg, and Wharton [10] and recently demonstrated by Junglen et al. [23]. By bending this guide into a torus it can be used as a storage ring $[10,24]$. Let us now consider what happens if the electrodes are of finite size. Along the $z$ axis the electric field will have a maximum at the center of the trap, therefore, molecules in high-field-seeking states will experience a (static) trapping potential along this direction. It is easily seen that at the center of the trap $\vec{\nabla} \cdot \vec{F}=0$. However, since $k_{z} \neq 0$ the defocusing force will be larger than the focusing force. When $k_{z}$ is not too large, this does not dramatically decrease the stability and molecules can be trapped in three dimensions. This trap is the neutral analog of the linear Paul trap for ions and was recently demonstrated for neutral molecules by Schnell et al. [25]. A microstructured version of this trap for neutral atoms was proposed and demonstrated by Katori et al. [26,27].

\section{B. Cylindrical ac trap}

Consider the geometry shown in Fig. 1(b). The structure consists of two ring electrodes and two cylindrically symmetric end caps. A negative voltage, $-\zeta$, is applied to the left end cap while an equal positive voltage, $\zeta$ is applied to the right end cap. The left ring electrode is kept at a positive voltage, $\xi$ while the right ring electrode is kept at an equal negative voltage $-\xi$. The electrostatic potential is antisymmetric about the $x-y$ plane and therefore, $\partial^{2} \Phi / \partial z^{2}=0$ everywhere on this plane. The electrostatic potential is symmetric about any plane containing the $z$ axis, and therefore $\partial \Phi / \partial x$ $=0$ and $\partial \Phi / \partial y=0$. Due to symmetry, $\partial^{2} \Phi / \partial x^{2}=\partial^{2} \Phi / \partial y^{2}$ at the $z$ axis, and from Laplace's equation both terms are zero at the point of intersection between the symmetry axis and the plane of antisymmetry, at the center of the trap. Again, in case of a linear or quadratic Stark shift, $\vec{\nabla} \cdot \vec{F}=0$ at the center of the trap [49]. The force constant along the $z$ axis is twice the force constant along $\rho, k_{z}=-2 k_{\rho}$, where $\rho=\sqrt{x^{2}+y^{2}}$. The size and direction of the force depend on the ratio of $\zeta$ to $\xi$. This ratio can be chosen such that $k_{z}=-2 k_{\rho}=a$ or $k_{z}=-2 k_{\rho}=$ $-a$, with $a$ a constant (see Sec. IV). In this case the electric field at the center of the trap is equal in magnitude and direction for both configurations. This trap is the neutral analog of the Paul trap for ions [19] and was first proposed by Peik [28]. This trap was recently demonstrated for neutral molecules by van Veldhoven et al. [6].

\section{Three-phase ac trap}

Consider the geometry shown in Fig. 1(c). The structure consists of six hemispherically ended electrodes that point toward the center of the trap. A positive voltage is applied to the right electrode and a negative voltage to the left electrode while the other electrodes are kept at ground potential [50]. Although the cylindrical symmetry is lost, the field has essentially the same form as the cylindrical ac trap discussed before. The main difference lies in the way the voltages are being switched. In the three-phase trap the voltages are alternately applied to the electrodes at the $x, y$, or $z$ axis. In this way, molecules will experience a focusing force $2 / 3$ of the 
(a)

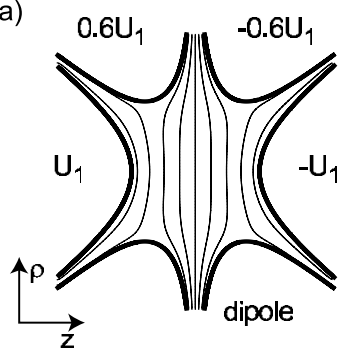

(c)

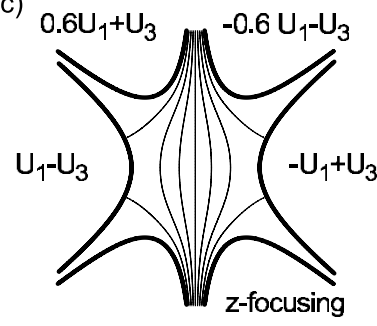

(b)

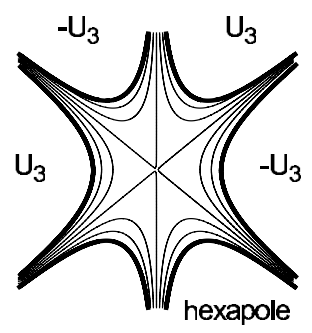

(d)

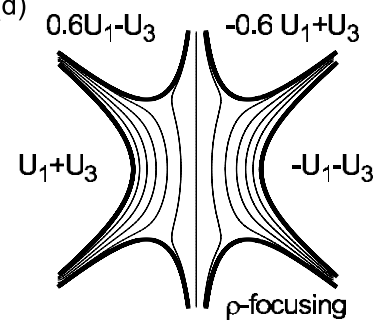

FIG. 2. Electrostatic potential obtained by applying different voltages to the electrodes of a cylindrical hexapole trap. (a) Dipole field. (b) Hexapole field. (c) $z$-focusing, obtained by subtracting a hexapole field from a dipole field. (d) $\rho$-focusing, obtained by adding a hexapole field to a dipole field. $U_{1}$ is taken to be equal to $3 U_{3}$.

time, while they experience a two-times-larger defocusing force $1 / 3$ of the time. This trap is the neutral analog of the three-phase trap for ions [29] and was proposed by Shimizu and Morinaga [30].

\section{OPTIMAL SHAPE}

In this section we will determine the optimal shape of the electrodes making up an ac trap. We will concentrate on the cylindrical ac trap which is used in the experiments to be described in Sec. VI. The optimal shape of the electrodes of a linear or three-phase ac trap can be determined in a similar fashion (see also [8]).

As we will see later, an ac trap has the largest depth and volume if the molecules experience a harmonic interaction potential. For molecules that experience a linear Stark shift the ideal form for the field strength is therefore also harmonic; $E(z, \rho)=E_{0}+\eta\left(z^{2}-\frac{1}{2} \rho^{2}\right)$, where the sign of $\eta$ can be reversed by switching the voltages.

We start by expanding the electric field in a Legendre series following [31]. In a region devoid of charges the electric field can be derived from the electric potential $\Phi$; $\vec{E}=-\vec{\nabla} \Phi$, with $\nabla^{2} \Phi=0$. $\Phi$ may be represented by a sum over spherical harmonics:

$$
\Phi=\sum_{L, M} a_{L M} r^{L} Y_{L M}
$$

Of most interest to us are terms with $M=0$, as these have cylindrical symmetry. Looking only at these terms, we write

$$
\Phi=\Phi_{0}+\Phi_{1} \frac{z}{z_{0}}+\Phi_{2} \frac{\left(z^{2}-\rho^{2} / 2\right)}{z_{0}^{2}}+\Phi_{3} \frac{\left(z^{3}-\frac{3}{2} z \rho^{2}\right)}{z_{0}^{3}} \cdots,
$$

with $z_{0}$ a scaling factor that characterizes the size of the electrode structure. The first term in Eq. (5) represents a constant voltage, the second term represents a constant electric field, and the third and fourth terms represent a quadrupole and hexapole trap, respectively. The voltage at $z=z_{0}$, $\rho=0$, is simply the sum over all coefficients, i.e., $\Phi\left(z=z_{0}, \rho\right.$ $=0)=\Sigma \Phi_{n}$ with the subscript $n$ denoting the different terms in Eq. (5). The electric field magnitude at the center is given by $E_{0}=\Phi_{1} / z_{0}$. As discussed in Sec. II, we require the magnitude of the electric field to be nonzero at the center of the trap, and symmetric under reflection in the $x-y$ plane and in any plane containing the $z$ axis. To achieve this we retain only terms of odd $n$ [51]. Anticipating the result that highorder terms introduce undesirable nonlinearities in the force we choose to retain only $\Phi_{1}, \Phi_{3}$, and $\Phi_{5}$. Hence

$$
\Phi=\Phi_{0}+\Phi_{1} \frac{z}{z_{0}}+\Phi_{3} \frac{\left(z^{3}-\frac{3}{2} z \rho^{2}\right)}{z_{0}^{3}}+\Phi_{5} \frac{\left(z^{5}-5 z^{3} \rho^{2}+\frac{15}{8} z \rho^{4}\right)}{z_{0}^{5}} .
$$

From this potential, we obtain the electric field magnitude, $E(z, \rho)=\sqrt{\left(\frac{\partial \Phi}{\partial z}\right)^{2}+\left(\frac{\partial \Phi}{\partial \rho}\right)^{2}}$. Throughout the region $z<z_{0}, \rho<z_{0}$ this can be expanded as a power series. For the case $\Phi_{5} \ll \Phi_{3} \ll \Phi_{1}$ we obtain

$$
\begin{aligned}
E(z, \rho)= & E_{0}\left[1+3\left(\frac{\Phi_{3}}{\Phi_{1}}\right) \frac{\left(z^{2}-\frac{1}{2} \rho^{2}\right)}{z_{0}^{2}}+\left(\frac{\Phi_{3}}{\Phi_{1}}\right)^{2} \frac{\left(\frac{9}{2} z^{2} \rho^{2}\right)}{z_{0}^{4}}\right. \\
& \left.+5\left(\frac{\Phi_{5}}{\Phi_{1}}\right) \frac{\left(z^{4}-3 z^{2} \rho^{2}+\frac{3}{8} \rho^{4}\right)}{z_{0}^{4}} \cdots\right] .
\end{aligned}
$$

The first two terms have the desired form and dominate the expansion. The other terms produce nonlinearities in the force, that, as we will see in Sec. VI, limit the trap depth. The field ideally contains only a $\Phi_{1}$ and $\Phi_{3}$ term.

Before looking at how we can create such a field we will first turn our attention to atoms and molecules that experience a quadratic Stark shift. In this case the electric field needs to be of the form $E^{2}(z, \rho)=E_{0}^{2}+\eta\left(z^{2}-\frac{1}{2} \rho^{2}\right)$. In a similar fashion we write $E^{2}$ in a power series as

$$
\begin{aligned}
E^{2}(z, \rho)= & E_{0}^{2}\left[1+6\left(\frac{\Phi_{3}}{\Phi_{1}}\right) \frac{\left(z^{2}-\frac{1}{2} \rho^{2}\right)}{z_{0}^{2}}+2\left(\frac{\Phi_{3}}{\Phi_{1}}\right)^{2} \frac{\left(\frac{9}{2} z^{2} \rho^{2}\right)}{z_{0}^{4}}\right. \\
& +9\left(\frac{\Phi_{3}}{\Phi_{1}}\right)^{2} \frac{\left(z^{2}-\frac{1}{2} \rho^{2}\right)^{2}}{z_{0}^{4}} \\
& \left.+10\left(\frac{\Phi_{5}}{\Phi_{1}}\right) \frac{\left(z^{4}-3 z^{2} \rho^{2}+\frac{3}{8} \rho^{4}\right)}{z_{0}^{4}} \cdots\right] .
\end{aligned}
$$

Again, we see that the field ideally contains only a $\Phi_{1}$ and $\Phi_{3}$ term. Compared to the case of a linear Stark shift, the potential contains extra terms in $\left(\Phi_{3} / \Phi_{1}\right)^{2}$, causing stronger deviations from an harmonic force.

In order to create the desired fields, we use the electrode configuration shown in Fig. 2. The surfaces of the electrodes, indicated by the bold curves in Fig. 2, are mapped onto the equipotentials of a cylindrical hexapole field. Consequently, when voltages of $U_{3}$ and $-U_{3}$ are applied alternately to the 

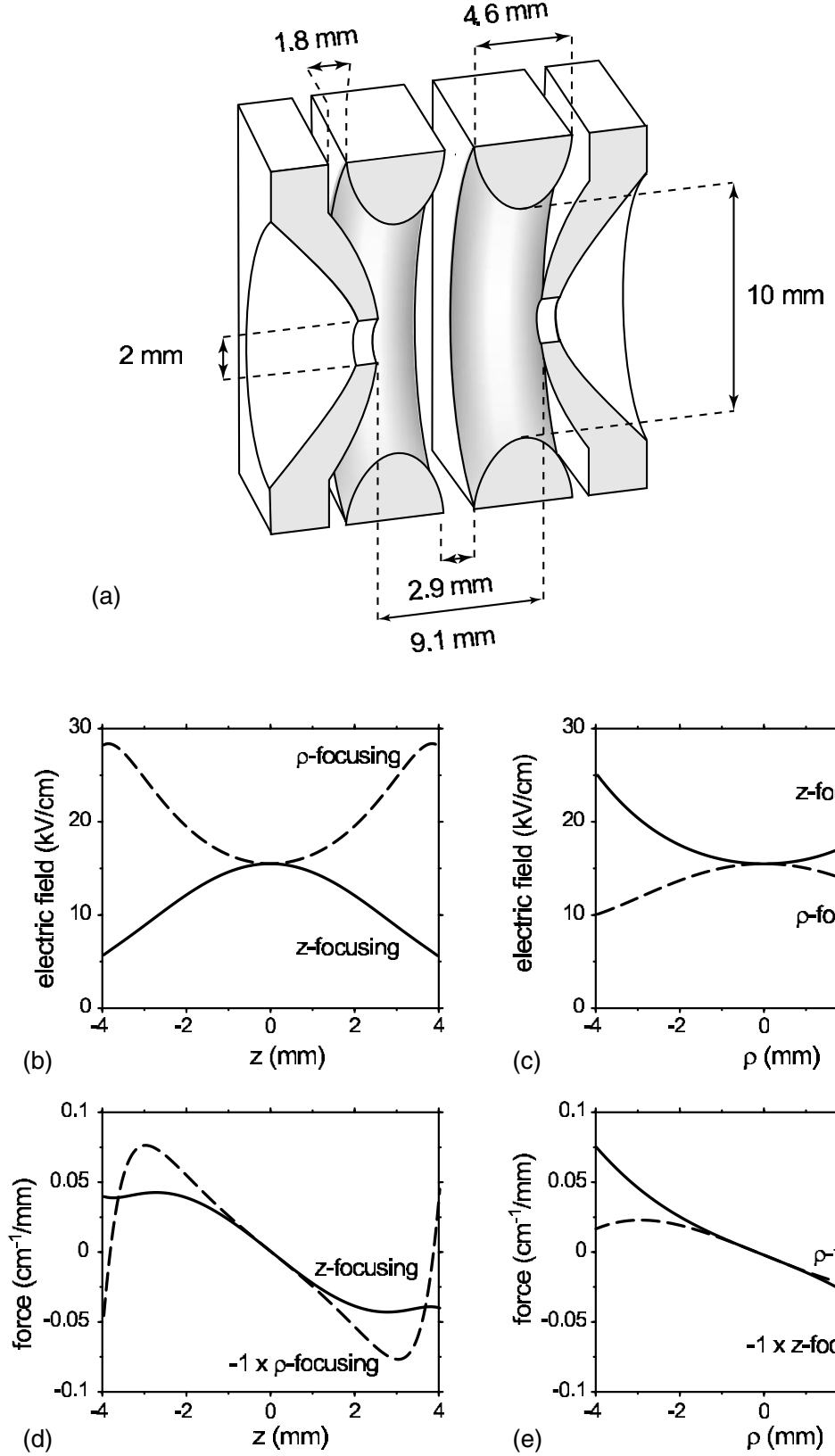
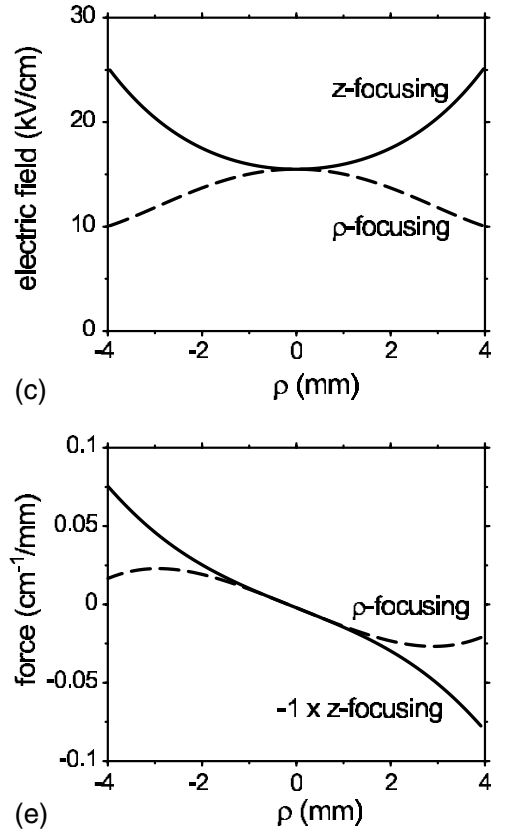

FIG. 3. (a) Schematic view of the cylindrical symmetric ac trap used in our experiments. (b) and (c) Electric field magnitude as function of the position along $z$ (b) and $\rho$ (c) when voltages of 5 , $7.5,-7.5$, and $-5 \mathrm{kV}$ (z-focusing) or $11,1.6,-1.6$, and $-11 \mathrm{kV}$ ( $\rho$-focusing) are applied to the electrodes. (d) and (e) Force on ammonia molecules in the highfield seeking component of the $|J, K\rangle=|1,1\rangle$ level along $z$ (d) and $\rho(\mathrm{e})$. four electrodes of the trap as shown in Fig. 2(b), a perfect hexapole field is obtained, i.e., $\Phi_{3}=U_{3}$, and $\Phi_{n}=0$, for $n$ $\neq 3$. In order to generate a dipole term, we apply voltages of $-U_{1},-0.6 U_{1}, 0.6 U_{1}$, and $U_{1}$ to the electrodes as shown in Fig. 2(a). Fitting this field to Eq. (5) yields $\Phi_{1}=0.88 U_{1}$, $\Phi_{3}=0$, and $\Phi_{5}=0.11 U_{1}$, with even terms being zero due to symmetry. For ac trapping, we apply a large dipole term and add (or subtract) a small time-varying hexapole field. Figure 2(c) shows the electrostatic potential when we subtract the hexapole field from the dipole field, with $U_{1}=3 U_{3}$. Along $z$, the electric field has a maximum at the center. Along $\rho$, the electric field has a minimum at the center. Molecules in high-field-seeking states will be focused along $z$ and defocused along $\rho$, and we will refer to this configuration as "z-focusing." Figure 2(d) shows the electrostatic potential when we add the hexapole field to the dipole field, again with $U_{1}=3 U_{3}$. Along $\rho$ the electric field has a maximum at the center. Along $z$, the electric field has a minimum at the center. Molecules in high-field-seeking states will be focused along $\rho$ and defocused along $z$, and we will refer to this configuration as " $\rho$-focusing."

The electric fields generated in this way contain a rather sizable $\Phi_{5}$ term that makes the force more nonlinear. Unfortunately, to decrease the $\Phi_{5}$ term present in the $z$-focusing field, we need to bring the electrodes closer together and increase the radii of the ring electrodes, whereas to decrease the $\Phi_{5}$ term present in the $\rho$-focusing field, we need to move the electrodes farther apart and decrease the radii of the ring electrodes. Improving the linearity of one configuration thus unavoidable results in deteriorating the linearity of the other. The use of a hexapole trap is a compromise, forced by the need to reverse the focusing and defocusing directions by adjusting the voltages only.

Figure 3(a) schematically shows the practical realization 
of our trap. The electrodes are truncated to avoid the distance between them becoming too small. The two end caps are placed a distance $2 z_{0}=9.1 \mathrm{~mm}$ apart. The two ring electrodes have a radius equal to $\sqrt{3 / 2} z_{0}=5 \mathrm{~mm}$. Both end caps have been given a hole with a $2 \mathrm{~mm}$ diameter to allow molecules to enter the trap and to extract ions that are produced in our laser-based detection scheme. In our experiment we use $U_{1}=8 \mathrm{kV}$ and $U_{3}=3 \mathrm{kV}$, resulting in voltages of $5,7.5$, -7.5 , and $-5 \mathrm{kV}$ (z-focusing) or $11,1.6,-1.6$, and $-11 \mathrm{kV}$ ( $\rho$-focusing) being applied to the electrodes. Fitting these fields to Eq. (5) yields $\Phi_{1}=7.0 \mathrm{kV}, \Phi_{3}=-3.0 \mathrm{kV}$, and $\Phi_{5}$ $=0.88 \mathrm{kV}$ ( $z$-focusing) and $\Phi_{1}=7.0 \mathrm{kV}, \Phi_{3}=3.0 \mathrm{kV}$, and $\Phi_{5}=0.62 \mathrm{kV}$ ( $\rho$-focusing). The resulting electric field magnitude as a function of the position along $z$ and $\rho$ is shown in Figs. 3(b) and 3(c), respectively. In the center of the trap the electric field magnitude is $16 \mathrm{kV} / \mathrm{cm}$. Figures $3(\mathrm{~d})$ and $3(\mathrm{e})$ show the corresponding force exerted on ammonia molecules in the high-field-seeking component of the $|J, K\rangle=|1,1\rangle$ level along the $z$ and $\rho$ axes, respectively. For ease of comparison the force in the $\rho$-focusing configuration in Fig. 3(d) and the force in the $z$-focusing configuration in Fig. 3(e) is multiplied by -1 . It is seen that the focusing and defocusing forces are equal at the center of the trap and that close to the center they are roughly linear, with the force constants given by

$$
\left|k_{\rho}\right|=\left|k_{z} / 2\right|=\frac{3 \mu_{\mathrm{eff}} \Phi_{3}}{z_{0}^{3}} .
$$

With $\mu_{\text {eff }}=0.0126 \mathrm{~cm}^{-1} /(\mathrm{kV} / \mathrm{cm})$ the effective dipole moment of ${ }^{15} \mathrm{ND}_{3}$, we find $\left|k_{z}\right|=0.024$ and $\left|k_{\rho}\right|$ $=0.012 \mathrm{~cm}^{-1} / \mathrm{mm}^{2}$. Further away from the center, the nonlinearity due to $\Phi_{5}$ acts to strengthen the defocusing power whereas the focusing is weakened. We will see later that this nonlinearity severely reduces the depth of the trap.

\section{MOTION IN THE TRAP}

In this section, the motion of molecules in the trap is investigated and the trap depth is determined as a function of the applied frequency. We start by assuming that the molecules experience a linear force that is alternately focusing and defocusing. Let us examine the motion along one of the principal axes of the trap, say $x$. The equation of motion can be written as

$$
m \frac{\partial^{2} x}{\partial t^{2}}+k(t) x=0
$$

with $m$ the mass of the molecule and $k(t)$ the force constant. We will apply a square wave voltage to the trap such that $k(t)$ is equal to $k$ during the time interval $\left(0, \frac{1}{2} T\right)$ and equal to $-k$ during the time interval $\left(\frac{1}{2} T, T\right)$. In this case Eq. (10) has a piecewise solution and is known as Hill's equation [32]. When $k$ is positive, the molecule will oscillate with a frequency $\Omega_{\mathrm{hex}}=\sqrt{|k| / m}$ around the center of the trap; $x(t)$ $=x\left(t_{0}\right) \cos \Omega_{\text {hex }}\left(t-t_{0}\right)+v_{x}\left(t_{0}\right) \Omega_{\text {hex }}^{-1} \sin \Omega_{\text {hex }}\left(t-t_{0}\right), \quad$ with $x\left(t_{0}\right)$ and $v_{x}\left(t_{0}\right)$ the initial position and velocity of the molecule, respectively. As the oscillation frequency is a function of the hexapole term we denote it with subscript "hex." When $k$ is negative, the amplitude will grow exponentially; $x(t)$ $=x\left(t_{0}\right) \cosh \Omega_{\text {hex }}\left(t-t_{0}\right)+v_{x}\left(t_{0}\right) \Omega_{\text {hex }}^{-1} \sinh \Omega_{\text {hex }}\left(t-t_{0}\right)$. The solution of the equation of motion is conveniently written in matrix form as

$$
\left(\begin{array}{c}
x(t) \\
v_{x}(t)
\end{array}\right)=M\left(t \mid t_{0}\right)\left(\begin{array}{c}
x\left(t_{0}\right) \\
v_{x}\left(t_{0}\right)
\end{array}\right)
$$

with the transfer matrix $M\left(t \mid t_{0}\right)$ given by

$$
M\left(t \mid t_{0}\right)=\left\{\begin{array}{cc}
\left(\begin{array}{cc}
\cos \Omega_{\mathrm{hex}}\left(t-t_{0}\right) & \Omega_{\mathrm{hex}}^{-1} \sin \Omega_{\mathrm{hex}}\left(t-t_{0}\right) \\
-\Omega_{\mathrm{hex}} \sin \Omega_{\mathrm{hex}}\left(t-t_{0}\right) & \cos \Omega_{\mathrm{hex}}\left(t-t_{0}\right)
\end{array}\right) & (F, \text { focusing force }), \\
\left(\begin{array}{cc}
\cosh \Omega_{\mathrm{hex}}\left(t-t_{0}\right) & \Omega_{\mathrm{hex}}^{-1} \sinh \Omega_{\mathrm{hex}}\left(t-t_{0}\right) \\
\Omega_{\mathrm{hex}} \sinh \Omega_{\mathrm{hex}}\left(t-t_{0}\right) & \cosh \Omega_{\mathrm{hex}}\left(t-t_{0}\right)
\end{array}\right) \quad(D, \text { defocusing force) } .
\end{array}\right.
$$

The transfer matrix is called $F$ when the force is focusing and $D$ when the force is defocusing.

The transfer matrix for any interval made up of subintervals is just the product of the transfer matrices of the subintervals:

$$
M\left(t_{2} \mid t_{0}\right)=M\left(t_{2} \mid t_{1}\right) M\left(t_{1} \mid t_{0}\right) .
$$

The transfer matrix for a single cycle is $F\left(T \mid \frac{1}{2} T\right) D\left(\frac{1}{2} T \mid 0\right)$. The transfer matrix for $N$ cycles is simply $M$ $=\left(F\left(T \mid \frac{1}{2} T\right) D\left(\frac{1}{2} T \mid 0\right)\right)^{N}$. In order for molecules to be stably trapped, it is necessary that all the elements of this transfer matrix remain bound when $N$ increases indefinitely. This is the case when $-1<\frac{1}{2} \operatorname{Tr}(M)<+1$ (see, for example, [33]).
It is useful to parametrize the transfer matrix for one cycle as [20]

$$
M(t+T \mid t)=\left(\begin{array}{cc}
\cos \Phi+\alpha \sin \Phi & \beta \sin \Phi \\
-\gamma \sin \Phi & \cos \Phi-\alpha \sin \Phi
\end{array}\right),
$$

where $\alpha(t), \beta(t)$, and $\gamma(t)$ are the Courant-Snyder parameters. It can be shown that the distribution of the molecules in phase-space is given by the Courant-Snyder phase-space ellipse:

$$
\gamma(t) x^{2}+2 \alpha(t) x v_{x}+\beta(t) v_{x}^{2}=\epsilon,
$$




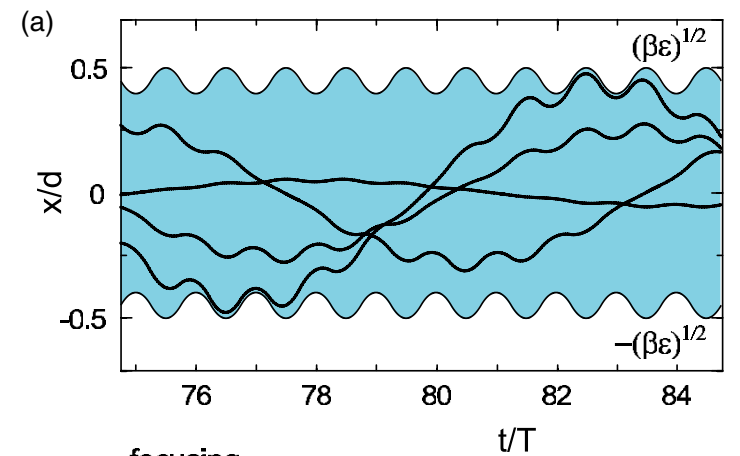

focusing

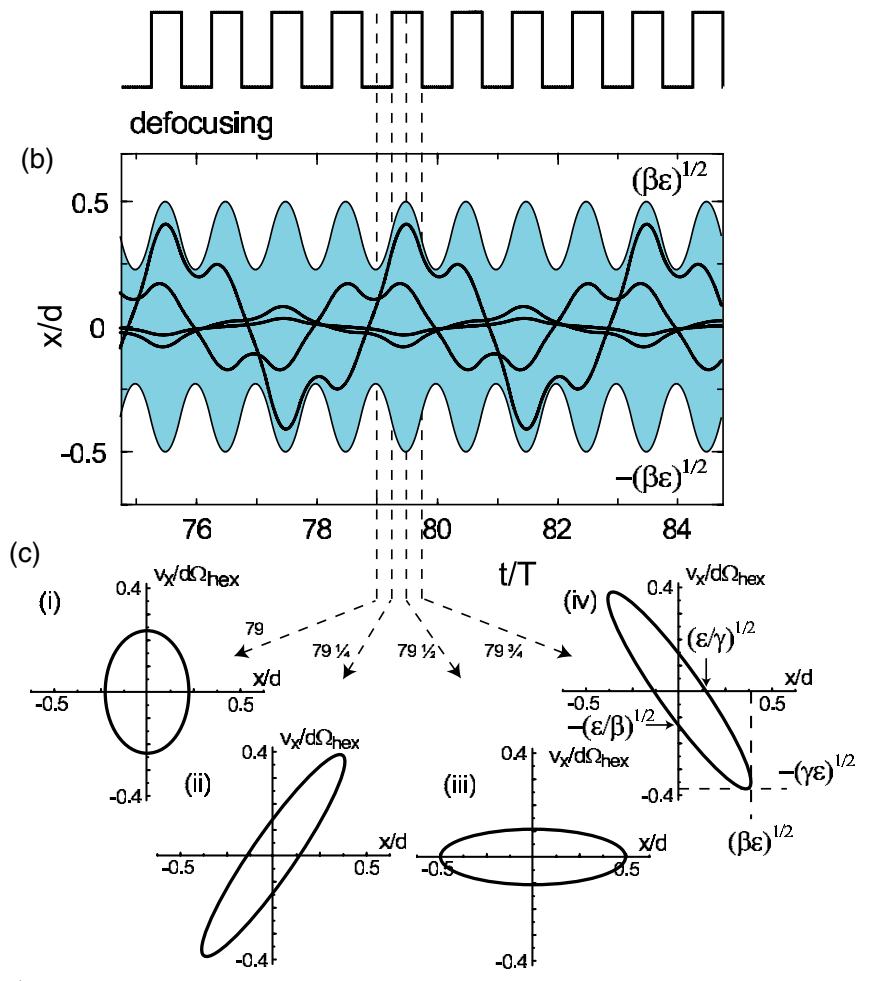

FIG. 4. (Color online) (a) Trajectories of ammonia molecules in an ac trap for $\Omega_{\text {driven }} / \Omega_{\text {hex }}=3.3$ corresponding to a phase advance of $\pi / 6$. The time is given in units of $T=2 \pi / \Omega_{\text {driven }}$, and the position is given in units of $d$, the distance between the trap electrodes along $x$. (b) As (a), but with $\Omega_{\text {driven }} / \Omega_{\text {hex }}=2$, corresponding to a phase advance of $\pi / 2$. (c) Phase-space area occupied by the trapped molecules at four times in a single cycle. The position is given in units of $d$ and the velocity in units of $d \Omega_{\mathrm{hex}}$.

with $\epsilon$ a constant which is called the emittance. The area enclosed by the Courant-Snyder ellipse is $\pi \epsilon$. The maximum position spread of a sample of trapped molecules with emittance $\epsilon$ is $\sqrt{\beta \epsilon}$. The maximum velocity spread of the trapped sample is $\sqrt{\gamma \epsilon}$. $\Phi$ is the phase advance per cycle, which is a measure for how far along the period a molecule has proceeded from its initial position. We see that the stability criterion is identical to requiring $\Phi$ to be real.

In Fig. 4 some trajectories in the trap are plotted as a function of time for two different values of the frequency of the applied voltage, $\Omega_{\text {driven }}$. The time is given in units of $T$ $=2 \pi / \Omega_{\text {driven, }}$, and the position is given in units of $d$, the distance between the trap electrodes along $x$. For the trajectories

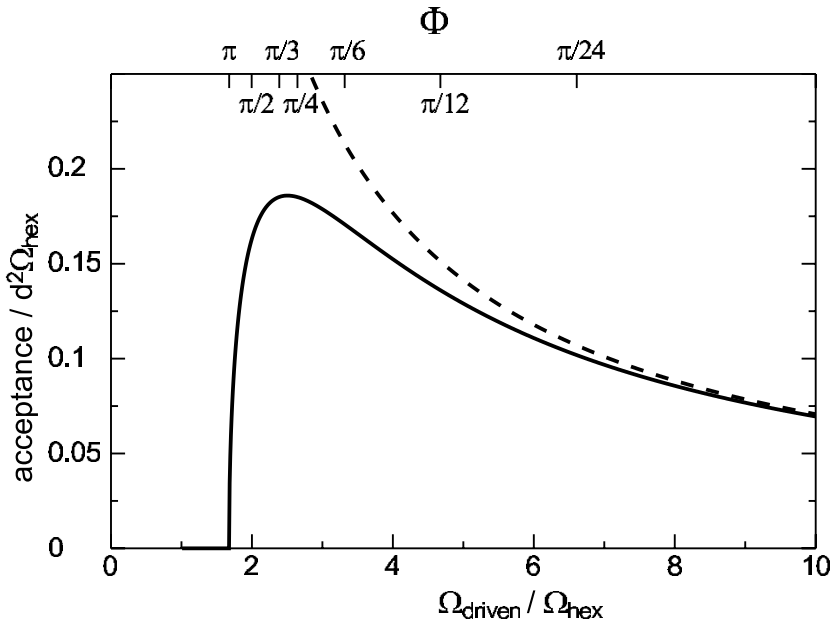

FIG. 5. The acceptance of the trap in units of $d^{2} \Omega_{\text {hex }}$ as a function of the applied frequency in units of $\Omega_{\text {hex }}$ calculated using the matrix method (solid line), and using an effective potential (dashed line). On the top the phase advance corresponding to some values of $\Omega_{\text {driven }} / \Omega_{\text {hex }}$ is shown.

shown in Fig. 5(a) $\Omega_{\text {driven }} / \Omega_{\text {hex }}=3.3$, which corresponds to a phase-advance of $\pi / 6$, implying that molecules return to their starting point after 12 cycles of the driving field. In the lower panel trajectories are shown for $\Omega_{\text {driven }} / \Omega_{\text {hex }}=2.0$, which corresponds to a phase-advance of $\pi / 2$, implying that molecules return to their starting point after four cycles of the driving field. The gray shaded area shows the size of the trapped cloud, bounded by $\pm \sqrt{\beta \epsilon}$. It can be seen that molecules are on average farther away from the center of the trap when the force is focusing than when the force is defocusing. This is the origin of the stability of the trap. At any fixed position, the divergence of the force averaged over one cycle, $(1 / T)(\partial / \partial x) \int_{0}^{T} F(x) d t$, is equal to zero. However, a molecule does not stay at a fixed position but will move toward the center of the trap under the influence of the focusing force. It will then be closer to the center, where the force is smaller, when the defocusing force is applied. This defocusing force will move the molecule farther away from the center again, bringing it in a region of a larger force when the focusing force is applied. As a consequence, the divergence of the force averaged over the trajectory of the molecule over one cycle, $(1 / T)(\partial / \partial x) \int_{0}^{T} F(x(t)) d t$, is less than zero. As this is a result of the motion of the molecule, this is commonly referred to as "dynamic" stability. For small values of $\Phi$, the motion can be separated in a rapid oscillationanalogous to the "micro motion" in ion traps [19]—at $\Omega_{\text {driven, }}$, the frequency of the applied fields, and a slower oscillation-analogous to the "macro" or "secular" motion in ion traps - at $\Omega_{\text {driven }} \Phi / 2 \pi$.

In Fig. 4(c) the phase-space area occupied by the trapped molecules is shown at four different phases of the driving field. This area is enclosed by the Courant-Snyder ellipse given by Eq. (15) and is equal to ( $\pi$ times) the emittance. The phase space ellipse rotates at the frequency of the applied field. Both the velocity spread and the position spread of the trapped cloud oscillate but the product of the two-the emittance-remains constant. We will use the largest emit- 
tance that can be accepted by the trap-the acceptance-as a measure for the trap depth.

The solid line in Fig. 5 shows the 1D acceptance calculated from the matrix model as a function of the frequency of the applied fields. The acceptance is given in units of $d^{2} \Omega_{\text {hex }}$. At low frequencies the trajectories of the molecules are unstable ( $\Phi$ is imaginary) and the acceptance is zero. Above $\Omega_{\text {driven }} / \Omega_{\text {hex }}=1.67$, corresponding to $\Phi=\pi$, the trap becomes abruptly stable. When the frequency is increased further, the molecules have less time to move in between switching times. Their amplitudes when the force is focusing and defocusing approach each other and the net force on the molecules averages out more [compare Figs. 4(a) and 4(b)]. As a result the acceptance decreases at higher frequencies. The highest acceptance is $0.186 d^{2} \Omega_{\text {hex }}$, obtained when $\Omega_{\text {driven }} / \Omega_{\text {hex }}=2.5$. This may be compared to a dc hexapole trap for low-field seekers which has an acceptance of $(\pi / 4) d^{2} \Omega_{\text {hex }}$; over four times larger.

It is instructive to compare the trap operated with a square wave by a trap operated with a sinusoidal function of the same amplitude, $k(t)=k \sin \Omega_{\text {driven }} t$. In this case Eq. (10) reduces to the well-known Mathieu equation [28,32]:

$$
\frac{d^{2} x}{d \tau^{2}}+\left(a-2 q_{x} \cos 2 \tau\right) x=0,
$$

with

$$
a=0, \quad q_{x}=\frac{2 \Omega_{\mathrm{hex}}^{2}}{\Omega_{\mathrm{driven}}^{2}}, \quad \tau=\frac{\Omega_{\mathrm{driven}} t}{2} .
$$

When $a=0$, solutions of the Mathieu equation are stable when $\left|q_{x}\right|<0.907$; i.e., when $\Omega_{\text {driven }} / \Omega_{\text {hex }}>1.48$. By comparing the acceptance found by numerically integrating Eq. (16) with the acceptance of the trap operated with a square wave, we find that the two are identical when $\Phi_{3}$ is multiplied by a factor 1.27 (and consequently $\Omega_{\text {hex }}$ is multiplied by a factor 1.12) when operating the trap with a square wave. This factor is equal to $4 / \pi$ and can be understood by expanding the square wave in a Fourier series. Molecules will mainly interact with the first term in the expansion, which has a Fourier coefficient equal to $4 / \pi$ [34]. This is similar to the description of the longitudinal motion in a Stark decelerator in terms of traveling waves [35].

At high frequencies, $\Omega_{\text {driven }} / \Omega_{\text {hex }} \gg 1$, the micromotion is much faster than the secular motion. In this limit, it is possible to derive a static "pseudo" potential, $\bar{W}[28,36,37]$. Again using the fact that, for a square wave, $\Omega_{\text {hex }}$ should be multiplied by $\sqrt{4 / \pi}$, we find

$$
\bar{W}=\frac{4}{\pi^{2}} m \frac{\Omega_{\text {hex }}^{4}}{\Omega_{\text {driven }}^{2}} x^{2} .
$$

The secular motion in this pseudopotential is an harmonic oscillation with frequency,

$$
\omega_{\text {secular }}=\frac{2 \sqrt{2}}{\pi} \frac{\Omega_{\text {hex }}^{2}}{\Omega_{\text {driven }}} .
$$

The acceptance of the pseudopotential is given by

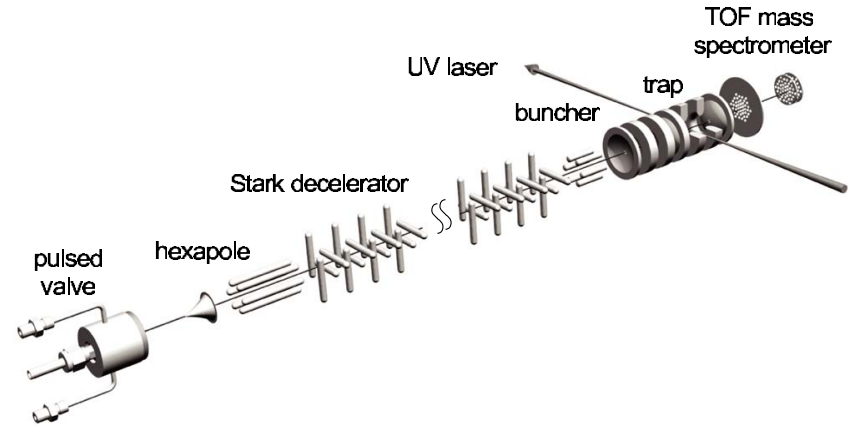

FIG. 6. Experimental setup. A molecular beam of ${ }^{15} \mathrm{ND}_{3}$ molecules in the low-field seeking component of the $|J, K\rangle=|1,1\rangle$ level is decelerated and loaded in the ac electric trap. Using a microwave pulse molecules are transferred to the high-field seeking state and subsequently trapped.

$$
\text { (acceptance) }=\frac{\pi}{4} d^{2} \omega_{\text {secular }}=\frac{1}{\sqrt{2}} d^{2} \frac{\Omega_{\mathrm{hex}}^{2}}{\Omega_{\mathrm{driven}}} .
$$

Equation (20) is shown as the dotted line in Fig. 5. We see that the acceptance can be reasonably well estimated from the pseudopotential for $\Omega_{\text {driven }} / \Omega_{\text {hex }}>7(q<0.04)$.

In our cylindrical trap, shown in Fig. 3, the hexapole frequencies along $z$ and $\rho$ are $\Omega_{\text {hex }, \mathrm{z}} / 2 \pi=590 \mathrm{~Hz}$ and $\Omega_{\text {hex }, \rho} / 2 \pi=417 \mathrm{~Hz}$, respectively. As a consequence, the phase advance along $z$ and $\rho$ is different at a given frequency. The trap can be made isotropic by switching the fields such that the configuration which focuses molecules along the $\rho$ direction is on for a longer time than the configuration that focuses molecules along the $z$ direction. We will define a duty cycle that is equal to $0 \%$ when $\rho$-focusing is continuously on, and equal to $100 \%$ when $z$-focusing is continuously on. In order to obtain an isotropic trap the duty cycle should be adjusted such that $\Phi_{z}=\Phi_{\rho}$ at each frequency. In the experiments presented in Sec. VI the duty cycle is modified either in this fashion or by using an approximated formula.

\section{TRAPPING AMMONIA MOLECULES IN A CYLINDRICAL AC TRAP}

The experimental setup used for ac trapping of ammonia molecules is depicted schematically in Fig. 6. It consists of two differentially pumped vacuum chambers separated by a $1 \mathrm{~mm}$ diameter skimmer. In the first chamber a molecular beam is made by expanding a mixture of $5 \%{ }^{15} \mathrm{ND}_{3}$ molecules seeded in xenon. The molecular beam is then passed through a Stark decelerator consisting of 95 electrode pairs. Molecules in the low-field-seeking component of the $|J, K\rangle$ $=|1,1\rangle$ level of ${ }^{15} \mathrm{ND}_{3}$ are decelerated to around $15 \mathrm{~m} / \mathrm{s}$. This part of the setup and the operation principle of the decelerator have been described in detail elsewhere $[38,39]$. The slow molecules exiting the decelerator are focused into the ac trap using a $12.5 \mathrm{~mm}$ long linear hexapole focuser and a cylindrical hexapole trap. The hexapole trap is almost identical to the AC trap depicted in Fig. 3. By applying a voltage of $5 \mathrm{kV}$ to the first ring electrode and the last end cap while keeping the other electrodes at ground an electric field is 
created that is zero at the center, and increases quadratically away from the center. By switching this field on and off when the molecules fly through it, molecules are focused along $\rho$ and bunched along the $z$ direction [39]. Using the linear hexapole focuser and cylindrical hexapole trap we make a 3D image of the packet exiting the Stark-decelerator at the center of the ac trap. For molecules having a linear Stark effect the force inside the linear and cylindrical hexapole is perfectly harmonic and the imaging can be performed without distortion. Due to the inversion splitting in ${ }^{15} \mathrm{ND}_{3}$, the force is nonlinear, causing the image to be distorted. As the acceptance of the trap is smaller than the phase-space volume of the beam exiting the decelerator (the emittance) most of the molecules that are lost would not be trapped anyway. When the packet of slow ammonia molecules enters the trap, we apply voltages of $7.5,5,0$, and $-11 \mathrm{kV}$ to the electrodes of the ac trap. This creates an electric field that is small at the entrance of the trap and increases toward the center of the trap. Molecules with a forward velocity of $15 \mathrm{~m} / \mathrm{s}$ will come to a standstill at the center of the trap. At that time, the high voltages on the trap are switched off and only the voltages used for extracting the ions remain. Using a $20 \mu$ s long microwave pulse the transition from the lowfield-seeking to the high-field-seeking hyperfine levels in the $|J, K\rangle=|1,1\rangle$ state of ${ }^{15} \mathrm{ND}_{3}$ can be induced. In zero electric field this transition is centered at $1.43 \mathrm{GHz}$ [38]. In the approximately $400 \mathrm{~V} / \mathrm{cm}$ extraction field, this transition is shifted to $1.46 \mathrm{GHz}$. The microwave radiation is generated by a Rohde\&Schwartz (SMR27) signal generator. Under optimum conditions, about $20 \%$ of the ammonia molecules are pumped to high-field-seeking levels. When the ac electric trap is switched on, the voltages on the trap are alternated at a frequency $\Omega_{\text {driven }}$ between the two configurations shown in Fig. 3. The frequency is generated by a function generator (Agilent 33220A) that triggers a total of eight high voltage switches (Behlke HTS201-03-GSM). The voltages are delivered by eight FUG (HCK400-20000) high voltage power supplies. After a certain trapping time, the trap is switched off, and the molecules are detected using pulsed UV-laser ionization followed by mass-selective detection of the parent ions. The $(2+1)$ resonance enhanced multi photon ionization scheme that is used selectively ionizes the ${ }^{15} \mathrm{ND}_{3}$ molecules in the upper or lower component of the $|J, K\rangle=|1,1\rangle$ inversion doublet, containing the low-field seeking or high-field seeking levels, respectively.

In Fig. 7 the ion signal is shown as a function of the switching frequency for molecules in low-field-seeking and high-field-seeking states. For clarity, the signal for molecules in low-field-seeking states has been given an offset. The signal for the high-field seekers is scaled up by a factor of 5, to correct for the $20 \%$ conversion efficiency in the microwave pumping process. The time the laser is fired is adjusted to be in phase with the switching frequency. Therefore, the time that the trap is on depends on the applied frequency but is always chosen to be close to $80 \mathrm{~ms}$. The measurements agree with the qualitative description of the dependence of the stability of the trap on $\Omega_{\text {driven }}$ as described in Sec. V. As we detect only molecules in the laser focus, our signal reflects the density at the center of the trap rather than the total number of molecules that are trapped. The signal is therefore

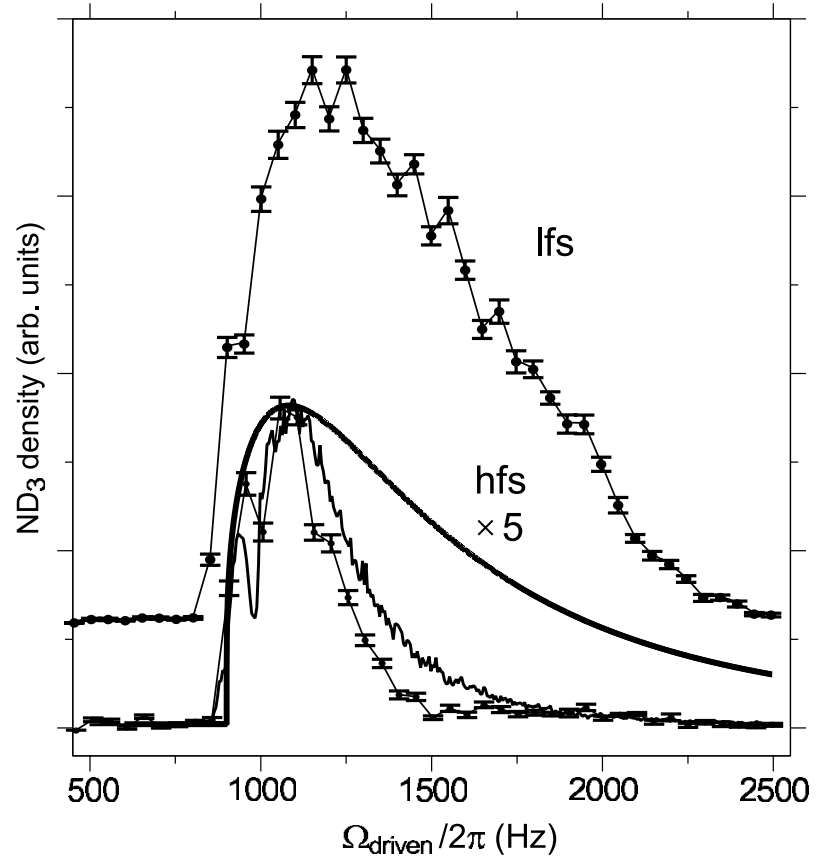

FIG. 7. Density of ${ }^{15} \mathrm{ND}_{3}$ molecules in low-field-seeking (lfs) and high-field seeking levels (hfs) of the $|J, K\rangle=|1,1\rangle$ state at the center of the trap as a function of the switching frequency, after the trap has been on for about $80 \mathrm{~ms}$. The signal of the high-field seekers is scaled up by a factor of 5 . The solid lines show the result of a linear model (bold) and a numerical simulation of the experiment using the true force (thin).

not proportional to the acceptance but rather proportional to $\sqrt{1 / \beta_{z}}\left(1 / \beta_{\rho}\right)$, with $\beta$ being the Courant-Snyder $\beta$ coefficient introduced in Sec. V (see Fig. 4). This function is shown as the bold line in Fig. 7. The thin line, also shown in Fig. 7, results from a numerical simulation of the experiment using the true (nonlinear) force on molecules. In the simulation the phase-space density in the trap is assumed to be homogeneously filled, i.e., it is assumed that the phase-space volume of the molecules exiting the decelerator is much larger than the acceptance of the trap. Both curves have been scaled to match the signal for high-field seekers at $\Omega_{\text {driven }} / 2 \pi$. The experimentally found cutoff frequency is around $900 \mathrm{~Hz}$, in good agreement with both the linear model and the simulation. Note that the cutoff frequency for the high-field seekers is slightly higher than for the low-field seekers due to an about $4 \%$ difference in the magnitude of $\mu_{\text {eff }}$ in the electric field at the center of the trap. With the present settings, the highest density of trapped molecules is observed at a switching frequency of $1100 \mathrm{~Hz}$, again in agreement with the calculations. At higher frequencies the signal decreases. The measured decrease is faster than predicted from the linear model. This is due to the higher-order terms in Eq. (7) giving rise to a (frequency independent) potential that lowers the trap depth for molecules in high-field-seeking states and increases the trap depth for molecules in low-field-seeking states. As seen, the experimentally observed density drops more rapidly than expected from the numerical simulation using the true force. We believe that the remaining difference is caused by a slight misalignment of our trap electrodes 

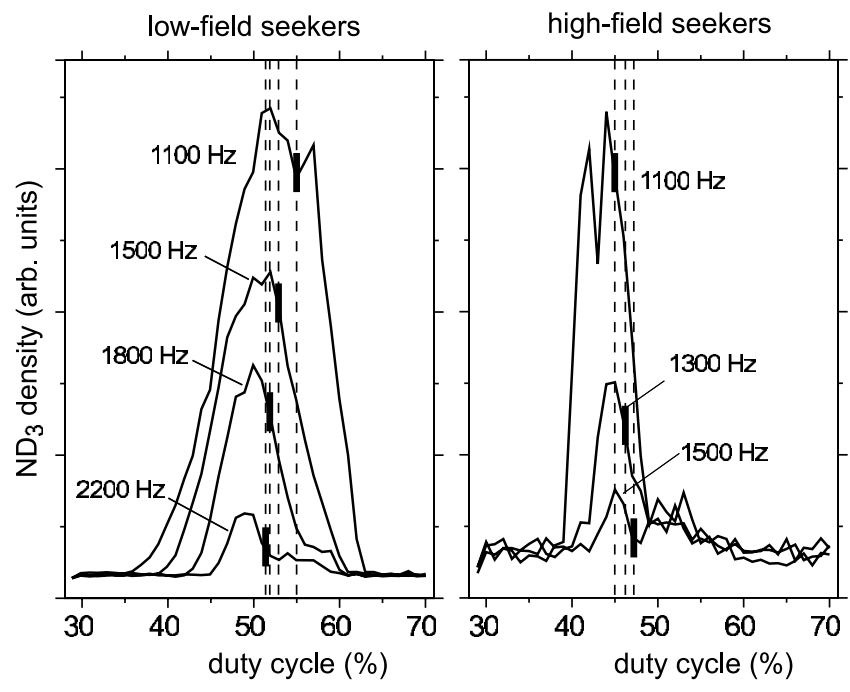

FIG. 8. Density of ${ }^{15} \mathrm{ND}_{3}$ molecules in the low-field-seeking (left column) and high-field-seeking state (right column) at the center of the trap as a function of the duty cycle for a number of different values of the applied frequency. The vertical lines show the value of the duty cycle for which the phase advance along $z$ and $\rho$ are equal.

(vide infra). This also explains the rapid decrease in signal of molecules in low-field-seeking states at higher frequencies. Note the dip in the measured and simulated curves around $1000 \mathrm{~Hz}$ due to nonlinear couplings that cause instabilities, also observed in ion traps [41].

Figure 8 shows the density at the center of the trap as a function of the duty cycle for a number of values of the frequency of the applied fields. The left column shows the measurements taken for molecules in low-field-seeking states and the right column those for molecules in high-fieldseeking states. As expected, the density is increased when the trap depth along $\rho$ is increased at the cost of a decrease of the trap depth along $z$. The vertical lines show the value of the duty cycle for which the phase advance along $z$ and $\rho$ are equal for the different frequencies. As can be seen, the vertical lines are always positioned at a duty cycle that is $3 \%$ higher than the maximum. We believe this is due to a slight misalignment of the electrodes which effectively adds an extra hexapole term-adding a force that is focusing along the $z$ direction and defocusing along $\rho$ for high-field-seeking states and vice versa for molecules in low-field-seeking states. This misalignment was also observed in an experiment on trapping molecules in a double-well potential performed in the same setup [40].

In our experiment, we use a tightly focused laser beam for detecting the molecules. This allows us to measure the spatial profile of the trapped molecules by scanning the position of the laser focus. Figure 9 shows measurements of the spatial distribution along the $z$ direction for molecules in highfield-seeking states. The trap is operated with a frequency of $\Omega_{\text {driven }} / 2 \pi=1100 \mathrm{~Hz}$ with a duty cycle equal to $45 \%$. The spatial distribution is recorded after 79 (lower) and $79 \frac{1}{2}$ (upper) cycles of the applied field, corresponding to two of the vertical lines in Fig. 4(b). The bold line shows the result of a Gaussian fit to the distribution. As expected, the packet is

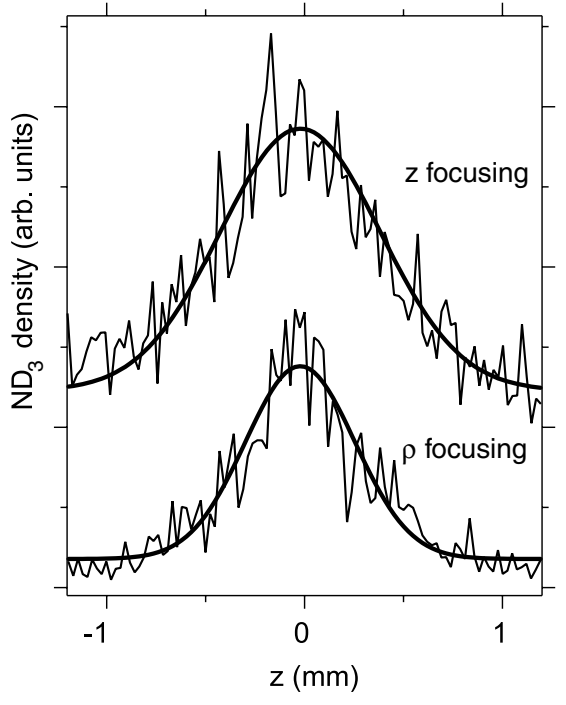

FIG. 9. Spatial distribution along $z$ of ${ }^{15} \mathrm{ND}_{3}$ molecules in highfield-seeking states after 79 (lower) and $79 \frac{1}{2}$ (upper) cycles of the applied field with $\Omega_{\text {driven }} / 2 \pi=1100 \mathrm{~Hz}$ and duty cycle $=45 \%$. The bold line shows a Gaussian fit to the distribution.

larger when the force is focusing along $z$ ( $z$-focusing) than when the force along $z$ is defocusing ( $\rho$-focusing). Interestingly, the ion signal at $z=0$ is roughly equal in both cases. When the width of the packet along $z$ is largest, the width along $\rho$ is smallest, i.e., the cloud oscillates between a cigarshaped distribution and a pancake-shaped distribution while the density at the center stays approximately constant.

Figure 10 shows the full width at half-maximum (FWHM) of the distribution of the trapped molecules as a function of the driving frequency. Shown are measurements after $79(\nabla)$ and $79 \frac{1}{2}(\triangle)$ cycles of the applied field, corresponding to $\rho$-focusing and $z$-focusing, respectively. In the linear model, the width of the packet along $z$ is proportional

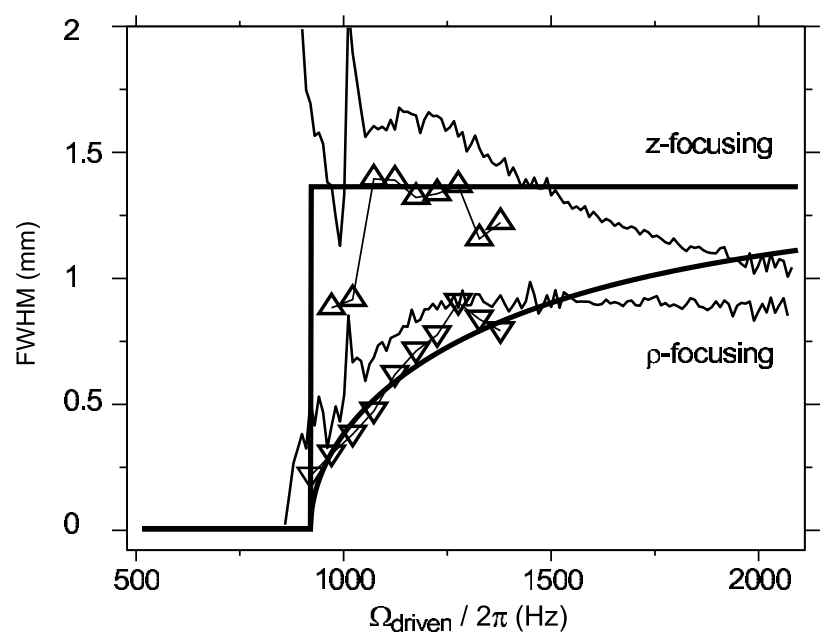

FIG. 10. FWHM of the spatial distribution of the trapped molecules as function of the drive frequency. The triangles show the measurements after $79(\nabla)$ and $79 \frac{1}{2}(\triangle)$ cycles of the applied field. The bold curves show the result of the matrix model, the thin curves show the result from a numerical simulation including the true force. 
to $\sqrt{\beta_{z}}$, with $\beta$ being the Courant-Snyder $\beta$ coefficient introduced in Sec. V (see Fig. 4). The bold curves in Fig. 10 show $\sqrt{\beta_{z}}$ as a function of $\Omega_{\text {driven }}$. The curves have been scaled to match the experimentally found width at $1100 \mathrm{~Hz}$. If the force is perfectly linear the width of the packet along $z$ at $z$-focusing is limited by the physical dimensions of the trap and is constant over the whole frequency range over which the trap is stable. In contrast, the width along $z$ at $\rho$-focusing depends strongly on the driving frequencies. At high frequency the width at $\rho$-focusing is equal to the width at $z$-focusing. At lower frequency the width of the packet along $z$ at $\rho$-focusing decreases until it becomes zero at the cutoff frequency. In our trap the width of the packet is determined by the nonlinearities in the force. The thin line also shown in Fig. 10 results from a numerical simulation using the true force. The numerical simulation is seen to reproduce the measured widths - both the absolute value and the frequency dependence-rather well. We attribute the $20 \%$ difference between the measured and calculated width to the slight misalignment of the trap electrodes discussed earlier. Around $\Omega_{\text {driven }} / 2 \pi=1000 \mathrm{~Hz}$, the simulated width is seen to oscillate. This is caused by the nonlinear resonance discussed earlier in connection to Fig. 7. At the resonance, the distribution of the trapped molecules at $z$-focusing is no longer correctly described by a single Gaussian profile. Rather, the distribution consists of a main peak with smaller side-peaks.

At high frequency, we can use the effective well model for predicting the width of the packet. For molecules in high field seeking states the nonlinear terms in Eq. (7) create a repulsive - frequency independent-potential. In order for molecules to stay trapped this potential must be balanced by the potential due to the ac force. Using Eqs. (7) and (18), we find the resulting potential, $W_{\text {secular }}$ :

$$
W_{\text {secular }}=\frac{4}{\pi^{2}} m \frac{\Omega_{\text {hex }, \mathrm{z}}^{4}}{\Omega_{\text {driven }}^{2}}-\mu_{\text {eff }} E_{0}\left(\frac{\Phi_{5}}{\Phi_{1}}\right) \frac{5 z^{4}}{z_{0}^{4}}
$$

where the duty cycle is set at $50 \%$ and terms going beyond $\Phi_{5}$ are ignored. The maximum extent of the packet is found by finding the position where the force on the molecules, $-\partial W_{\text {secular }} / \partial z$, becomes equal to zero:

$$
z_{\max }=\sqrt{\frac{12}{5}} \frac{1}{\pi} \sqrt{\frac{\Phi_{3}}{\Phi_{5}}} \frac{\Omega_{\mathrm{hex}, \mathrm{z}}}{\Omega_{\mathrm{driven}}} z_{0} .
$$

From Eq. (22), we find that the width of the spatial profile decreases as $1 / \Omega_{\text {driven, in agreement with the numerical }}$ simulations shown in Fig. 10. At $\Omega_{\text {driven }} / 2 \pi=2000 \mathrm{~Hz}$, we find from Eq. (22) $z_{\max }=1.2 \mathrm{~mm}$. When the duty cycle as used in our experiment is taken into account, we find $z_{\max }$ $=0.9 \mathrm{~mm}$. This is the maximum extent of the packet and can therefore not be compared directly to the FWHM plotted in Fig. 10. From numerical simulation we find $z_{\max }=0.8 \mathrm{~mm}$.

We can determine the velocity distribution of the trapped molecules by measuring how rapidly the cloud expands after the trap has been switched off. In Fig. 11 the spatial distributions of ${ }^{15} \mathrm{ND}_{3}$ molecules in high-field-seeking states are shown after an expansion time that is indicated in the figure. The molecules are released after 79 cycles of the applied field with $\Omega_{\text {driven }} / 2 \pi=1100 \mathrm{~Hz}$ and duty cycle $=45 \%$. The

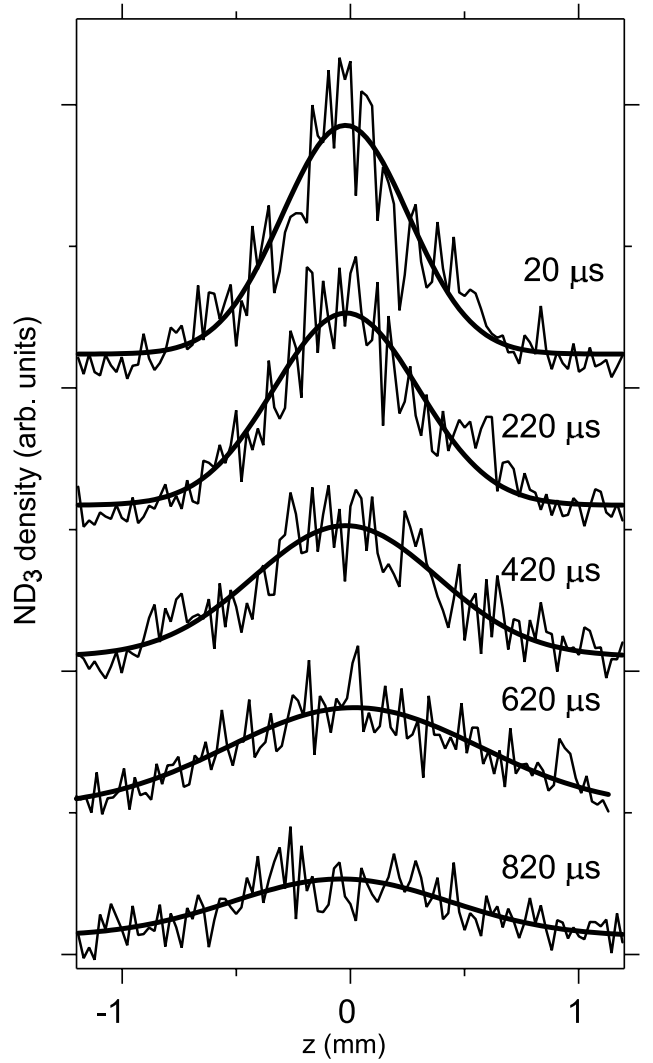

FIG. 11. Spatial distributions of ${ }^{15} \mathrm{ND}_{3}$ molecules in high-fieldseeking states after having expanded for a time indicated in the figure. The molecules are released from the trap after 79 cycles of the applied field with $\Omega_{\text {driven }} / 2 \pi=1100 \mathrm{~Hz}$ and duty cycle $=45 \%$. The bold line shows the result of a Gaussian fit to the distribution.

bold line shows the result of a Gaussian fit to the distribution. Similar measurements were taken for molecules released after $79 \frac{1}{4}, 79 \frac{1}{2}$, and $79 \frac{3}{4}$ cycles.

In Fig. 12 the FWHM that result from a Gaussian fit to the spatial distribution are shown as a function of the time after the molecules have been released from the trap. The trap is switched off after $79(\nabla), 79 \frac{1}{4}(+), 79 \frac{1}{2}(\triangle)$, or $79 \frac{3}{4}(\times)$ cycles of the applied field with $\Omega_{\text {driven }} / 2 \pi=1100 \mathrm{~Hz}$ and duty cycle $=45 \%$. These times correspond to those shown as the vertical lines in Fig. 4. As expected, if we release the molecules after 79 cycles of the applied field-when the field is focusing along $\rho$-the cloud starts off small, but expands rapidly. If we release the molecules after $79 \frac{1}{2}$ cycles of the applied field - when the field is focusing along $z$ - the clouds starts off larger but expands less rapidly. After $79 \frac{1}{4}$ and $79 \frac{3}{4}$ cycles of the applied field the phase-space distribution is tilted with respect to the position and velocity axes. In both cases the velocity spread is larger than the velocity spread after 79 cycles of the applied field as is apparent from Fig. 4. Interestingly, after $79 \frac{3}{4}$ cycles the phase-space distribution is tilted such that all molecules have a velocity toward the center of the trap along the $z$ axis of the trap. Therefore, at first the width along $z$ becomes smaller and comes to a minimum about $250 \mu$ s after the trap is switched off.

If we assume the velocity distribution to be Gaussian, the cloud expands as 


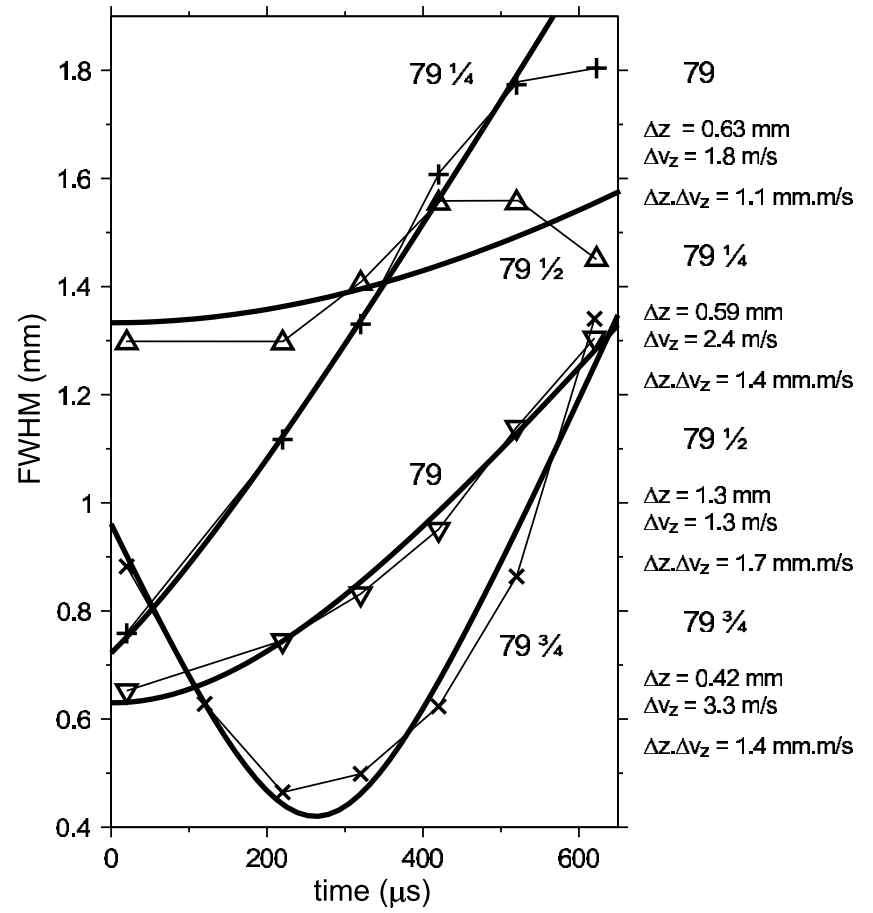

FIG. 12. The FWHM that result from a Gaussian fit to the spatial distribution as a function of the expansion time. The molecules are released from the trap after $79(\nabla), 79 \frac{1}{4}(+), 79 \frac{1}{2}(\triangle)$, or $79 \frac{3}{4}(\times)$ cycles of the applied field with $\Omega_{\text {driven }} / 2 \pi=1100 \mathrm{~Hz}$ and duty cycle $=45 \%$. The bold curves show a square root formula fitted to the data.

$$
\Delta z(t)=\sqrt{(\Delta z(t=0))^{2}+\left(\Delta v_{z} t\right)^{2}}
$$

with $\Delta z(t=0)$ the initial spatial distribution of the trapped molecules and $\Delta v_{z}$ the velocity distribution. The bold curves show the fitted formula to the data. The fitted values for the velocity spread and the initial position spread are given in the figure. As the area of the phase-space distribution stays constant we expect $\Delta z(t=0)$ times $\Delta v_{z}$ to be a constant. In actual effect, we find that the measured areas differ by more than $50 \%$. Note that the determined velocity spread after 79 cycles of the applied field is more precise than the one measured after $79 \frac{1}{2}$ cycles. After 79 cycles the cloud expands rapidly along $z$ and much more slowly along the $\rho$ direction. Therefore, the signal integrated over $z$ stays nearly constant. After $79 \frac{1}{2}$ cycles the cloud expands rapidly along $\rho$ and much more slowly along the $z$ direction. The signal integrated over $z$, therefore, drops rather quickly.

Figure 13 shows the density at the center of the trap as a function of time for molecules in low-field-seeking (upper curve) and high-field-seeking states (lower curve). The bold lines through the data show an exponential fit. Molecules are seen to escape the trap with a $1 / e$ time of $0.17 \mathrm{~s}$ for low-field seekers and $0.18 \mathrm{~s}$ for high-field seekers. Within the error bars these lifetimes are equal to one another and to the $0.17 \mathrm{~s}$

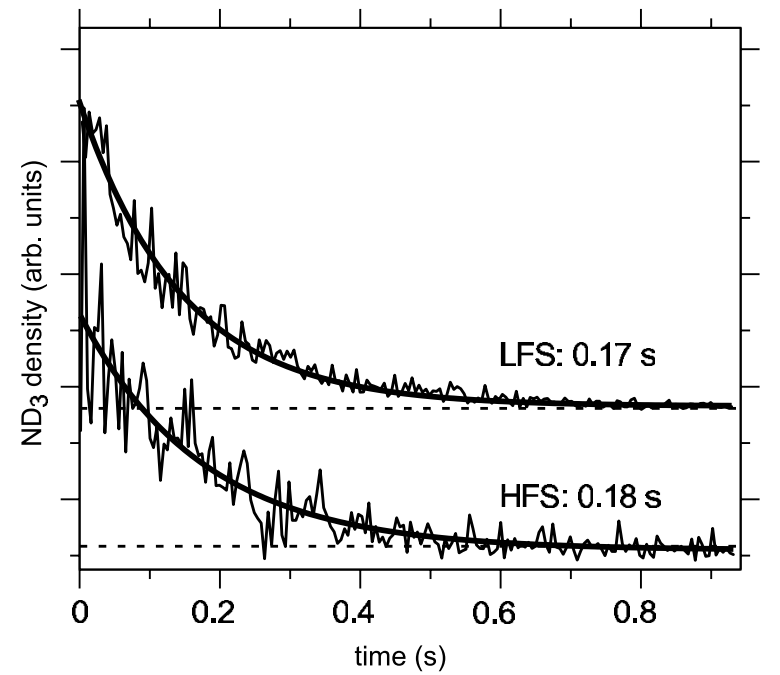

FIG. 13. Density of ${ }^{15} \mathrm{ND}_{3}$ molecules in low-field-seeking (upper curve) and high-field-seeking states (lower curve) at the center of the trap as a function of time. The bold lines show an exponential fit to the data with $1 / e=0.17 \mathrm{~s}$ (low-field seekers) and $1 / e=0.18 \mathrm{~s}$ (high-field seekers).

found in earlier experiments performed in the same setup on molecules in low-field seeking states trapped in a static trap [40]. We therefore conclude that the dominant loss channel is due to collisions with background atoms in the relatively poor $\left(P=5 \times 10^{-8}\right.$ torr $)$ vacuum.

At early times immediately after the trap is switched on, the density at the center of the trap shows some slight oscillations. These are mainly due to molecules that do not remain trapped. In order to measure the trap frequency of the molecules directly, we have tried to make these oscillations more pronounced by deliberately switching the trap on too early, or by switching the trap off temporarily after having been on for $80 \mathrm{~ms}$. Although these attempts were unsuccessful, we were able to verify that the trap frequency of the molecules along $z, \omega_{\text {secular, }} / 2 \pi$, is equal to about $240 \mathrm{~Hz}$, when we apply a frequency $\Omega_{\text {driven }} / 2 \pi=1100 \mathrm{~Hz}$ with a duty cycle of $45 \%$, as expected from our simulations.

In all experiments discussed so far, we switch instantaneously (within $200 \mathrm{~ns}$ ) between the two different trap configurations. Under some conditions the trap will still be stable if we leave it off for a certain time before switching to the next configuration. During this time the motion of the molecules is $x(t)=x\left(t_{0}\right)+v_{x}\left(t_{0}\right)\left(t-t_{0}\right)$, with $x\left(t_{0}\right)$ and $v_{x}\left(t_{0}\right)$ the initial position and velocity of the molecule, respectively. So the transfer matrix is simply:

$$
M\left(t \mid t_{0}\right)=\left(\begin{array}{cc}
1 & t-t_{0} \\
0 & 1
\end{array}\right) \quad(O, \text { free flight })
$$

The transfer matrix during free flight is written as $O$. The transfer matrix for a single cycle now becomes

$$
F\left(T_{\text {on }}+T_{\text {off }} \mid \frac{1}{2} T_{\text {on }}+T_{\text {off }}\right) O\left(\frac{1}{2} T_{\text {on }}+T_{\text {off }} \mid \frac{1}{2} T_{\text {on }}+\frac{1}{2} T_{\text {off }}\right) D\left(\frac{1}{2} T_{\text {on }}+\frac{1}{2} T_{\text {off }} \mid \frac{1}{2} T_{\text {off }}\right) O\left(\frac{1}{2} T_{\text {off }} \mid 0\right)
$$




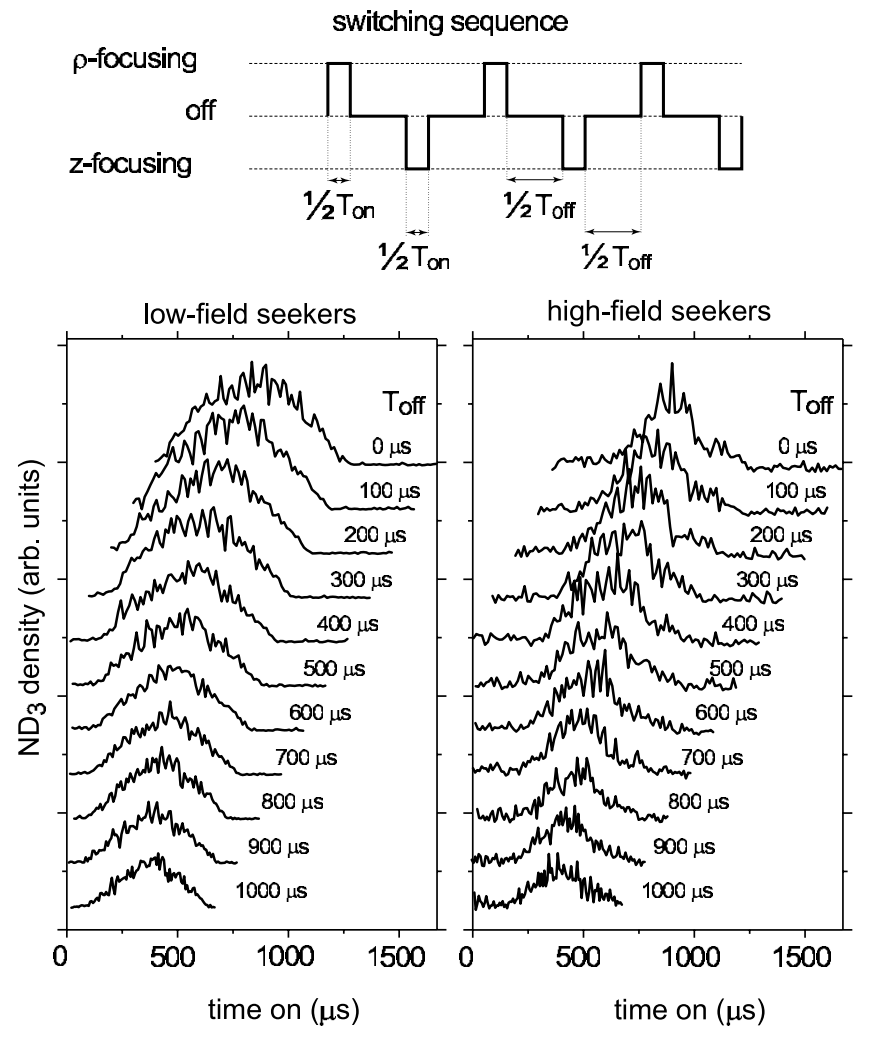

FIG. 14. Density of ${ }^{15} \mathrm{ND}_{3}$ molecules in low-field-seeking (left column) and high-field-seeking states (right column) at the center of the trap as function of the time that the focusing and defocusing fields are on for a number of different values for which the fields are off.

The stability for this arrangement can be determined as before. Figure 3 of [8] shows the stability diagram for an alternate gradient decelerator. This can be translated to our situation by simply replacing $\kappa L$ and $\kappa S$ by $\Omega_{\text {hex }} T_{\text {on }}$ and $\Omega_{\text {hex }} T_{\text {off }}$, respectively.

Figure 14 shows the density at the center of the trap as a function of $T_{\text {on }}$ for a number of different values of $T_{\text {off }}$. The measurements on the left are for molecules in low-fieldseeking states, the measurements on the right for molecules in high-field-seeking states. As before, the duty cycle is adjusted such that the phase advance along $z$ is equal to the phase advance along $\rho$. The measurements taken with $T_{\text {off }}$ $=0$ are identical to those shown in Fig. 7 but now plotted as function of $2 \pi / \Omega_{\text {driven. }}$. When $T_{\text {off }}$ is increased, the maximum of the curves shifts to smaller values of $T_{\text {on }}$. Interestingly, the maximum density drops only slightly for higher values of $T_{\text {off }}$. When the fields are switched off for $500 \mu \mathrm{s}$, the maximum density is obtained for $T_{\text {on }}=500 \mu$ s, i.e., the fields are applied only half of the time. With these settings, the density at the center of the trap is decreased by less than $20 \%$ compared to the situation that the fields are applied all the time.

\section{SUMMARY AND CONCLUSIONS}

In this paper, we have experimentally demonstrated trapping of molecules in both high-field-seeking and low-fieldseeking states in a cylindrically symmetric electric ac trap.
We have measured the spatial profile of the trapped cloud immediately after release and after a variable expansion time. We have compared these measurements to a simple linear model and to numerical simulations taking into account the true force on the molecules. By changing the duty cycle of the square wave voltage that is applied to the trap, the trapping frequencies along $z$ and $\rho$ can be chosen to be the same. At $1100 \mathrm{~Hz}$ and a duty cycle of $45 \%$ the trapping frequency is around $240 \mathrm{~Hz}$. With these settings we find the FWHM to be $\Delta z=1.3 \mathrm{~mm}, \Delta v_{z}=1.3 \mathrm{~m} / \mathrm{s}(T=0.8 \mathrm{mK})$ and $\Delta z=0.63 \mathrm{~mm}, \Delta v_{z}=1.8 \mathrm{~m} / \mathrm{s}(T=1.5 \mathrm{mK})$ when the fields are focusing along $z$ and $\rho$, respectively. From numerical simulations, we find the phase-space volume that can be confined by the trap (the acceptance) to be $270 \mathrm{~mm}^{3}(\mathrm{~m} / \mathrm{s})^{3}$, corresponding to a trap depth of about $5 \mathrm{mK}$ and a trap volume of about $20 \mathrm{~mm}^{2}$. The phase-space volume (the emittance) of the Stark decelerated molecular beam used to load the trap is about five times larger than the acceptance of the trap [39]. Therefore, we expect to homogeneously fill the acceptance of the trap, which is consistent with our measurements.

We find-both from simulation and from measurements - that the acceptance is limited by the nonlinearities of the forces. Due to these nonlinearities the maximum position spread of the packet scales as $1 / \Omega_{\text {driven }}^{3}$, and consequently, the acceptance scales as $1 / \Omega_{\text {driven }}^{9}$ rather than the expected $1 / \Omega_{\text {driven }}^{3}$ if the forces would be perfectly linear. This has important consequences for the possibility of using evaporative - and to a lesser extent-sympathetic cooling in ac traps. When collisions between trapped molecules occur, energy that is stored in the micro motion can be transferred into secular motion. This process is called "viscous" heating. Viscous heating competes with the "good" consequences of collisions-thermalization and evaporation of the hottest molecules over the trap barrier leading to cooling. As the amount of viscous heating scales as $\left(\omega_{\text {secular }} / \Omega_{\text {driven }}\right)^{2}$, evaporative cooling is more likely to work at higher frequencies $[22,42]$.

In minimizing nonlinearities, we are constrained by the need to reverse the focusing and defocusing directions by adjusting the voltages only. When the shape of the electrodes is modified to improve the linearity of the focusing force in one configuration, the linearity of the other configuration is deteriorated. It was argued in Sec. IV that the cylindrical hexapole geometry used in our experiments has in fact the optimal shape. Other possible electrode geometries suffer from the same problem. Using the experimentally validated program used for the cylindrical trap, we have also calculated the acceptance for the linear and three-phase ac trap presented in Sec. III and found them to be comparable.

Besides the acceptance and strength of confinement, there are other important criteria to choose a specific ac trap. The main advantage of the cylindrical trap over the other two traps is that at the center the electric field has a (controllable) constant direction and magnitude. This may be important for collision studies and experiments aimed at detecting the permanent electric dipole moment of the electron. Moreover, this may be needed for avoiding Majorana transitions induced by the varying electric field. As demonstrated in this paper the electric fields of the trap can also be repeatedly 
switched off - or switched to a variable homogeneous electric field-for as long as half a millisecond without significantly decreasing the trap depth. The cylindrical and threephase trap can be used for ac trapping molecules in both high-field-seeking and low-field-seeking states. Furthermore, by applying the appropriate voltages, the cylindrical symmetric trap can be used for trapping molecules in low-fieldseeking states in a static quadrupole or hexapole potential [40], and the three-phase trap can be used for trapping molecules in low-field-seeking states in a Ioffe-Pritchard trap $[43,44]$. The main advantages of the linear and, to a lesser extent, the three-phase trap are that they are conveniently loaded from a Stark decelerator [25], and are very accessible for laser beams to detect and manipulate the trapped molecules.

It is useful to compare the ac traps with other traps for neutral molecules that have been demonstrated. Traps using inhomogeneous static electric and magnetic fields are easily $1 \mathrm{~K}$ deep and typically have a trap volume of $1 \mathrm{~cm}^{3}$ [1-4].
These traps can only be used to trap molecules in low-fieldseeking states. Alhough it is impossible to create a field maximum using static electric or magnetic field, this does not apply to optical [45] or microwave fields [46]. Molecules in high-field-seeking states have been trapped in the focus of a $\mathrm{CO}_{2}$ laser beam [5]. The light traps thus far demonstrated have typically a trap depth of a few hundred $\mu \mathrm{K}$ and a volume of $10^{-5} \mathrm{~cm}^{3}$. Our ac electric trap demonstrated here can be used to trap molecules in both low-field- and high-fieldseeking states and has a trap depth of about $5 \mathrm{mK}$ and a trap volume of about $20 \mathrm{~mm}^{3}$.

\section{ACKNOWLEDGMENTS}

We acknowledge the expert technical assistance of A.J.A. van Roij and H. Haak. We thank B. Friedrich, P. Lützow, A. Marian, S. Schlunk, M.R. Tarbutt, and E.A. Hinds for helpful discussions.
[1] J. D. Weinstein, R. deCarvalho, T. Guillet, B. Friedrich, and J. M. Doyle, Nature (London) 395, 148 (1998).

[2] N. Vanhaecke, W. de Souza Melo, B. L. Tolra, D. Comparat, and P. Pillet, Phys. Rev. Lett. 89, 063001 (2002).

[3] H. L. Bethlem, G. Berden, F. M. H. Crompvoets, R. T. Jongma, A. J. A. van Roij, and G. Meijer, Nature (London) 406, 491 (2000).

[4] S. Y. T. van de Meerakker, P. H. M. Smeets, N. Vanhaecke, R. T. Jongma, and G. Meijer, Phys. Rev. Lett. 94, 023004 (2005).

[5] T. Takekoshi, B. M. Patterson, and R. J. Knize, Phys. Rev. Lett. 81, 5105 (1998).

[6] J. van Veldhoven, H. L. Bethlem, and G. Meijer, Phys. Rev. Lett. 94, 083001 (2005).

[7] J. L. Bohn, Phys. Rev. A 63, 052714 (2001).

[8] H. L. Bethlem, M. R. Tarbutt, J. Küpper, D. Carty, K. Wohlfart, E. A. Hinds, and G. Meijer, J. Phys. B 39, R263 (2006).

[9] J. Jeans, The Mathematical Theory of Electricity amd Magnetism (Cambridge University Press, London, 1960).

[10] D. Auerbach, E. E. A. Bromberg, and L. Wharton, J. Chem. Phys. 45, 2160 (1966).

[11] W. H. Wing, Prog. Quantum Electron. 8, 181 (1984).

[12] W. Ketterle and D. E. Pritchard, Appl. Phys. B: Photophys. Laser Chem. 54, 403 (1992).

[13] J. J. Livingood, Principles of Cyclic Particle Accelerators (van Nostrand, New Jersey, 1961).

[14] J. C. Helmer, F. B. Jacobus, and P. A. Sturrock, J. Appl. Phys. 31, 458 (1960).

[15] K. R. Chien, P. B. Foreman, K. H. Castleton, and S. G. Kukolich, Chem. Phys. 7, 161 (1975).

[16] H. J. Loesch and B. Scheel, Phys. Rev. Lett. 85, 2709 (2000).

[17] S. K. Sekatskiǔ, JETP Lett. 62, 916 (1995).

[18] R. T. Jongma, G. von Helden, G. Berden, and G. Meijer, Chem. Phys. Lett. 270, 304 (1997).

[19] W. Paul, Rev. Mod. Phys. 62, 531 (1990).

[20] E. D. Courant and H. S. Snyder, Phys. Rev. 88, 1190 (1952).

[21] R. V. E. Lovelace, C. Mehanian, T. J. Tommila, and D. M. Lee,
Nature (London) 318, 30 (1985).

[22] E. A. Cornell, C. Monroe, and C. E. Wieman, Phys. Rev. Lett. 67, 2439 (1991).

[23] T. Junglen, T. Rieger, S. A. Rangwala, P. W. H. Pinkse, and G. Rempe, Phys. Rev. Lett. 92, 223001 (2004).

[24] H. Nishimura, G. Lambertson, J. G. Kalnins, and H. Gould, Eur. Phys. J. D 31, 359 (2004).

[25] Schnell et al., in preparation.

[26] H. Katori and T. Akatsuka, Jpn. J. Appl. Phys., Part 1 43, 358 (2004).

[27] T. Kishimoto, H. Hachisu, J. Fujiki, K. Nagato, M. Yasuda, and H. Katori, Phys. Rev. Lett. 96, 123001 (2006).

[28] E. Peik, Eur. Phys. J. D 6, 179 (1999).

[29] R. F. Würker, H. M. Goldenberg, and R. V. Langmuir, J. Appl. Phys. 30, 441 (1959).

[30] F. Shimizu and M. Morinaga, Jpn. J. Appl. Phys., Part 1 31, L1721 (1992).

[31] T. Bergeman, G. Erez, and H. J. Metcalf, Phys. Rev. A 35, 1535 (1987).

[32] M. Abramowitz and I. A. Stegun, Handbook of Mathematical Functions, 9th ed. (Dover, New York, NY, 1970).

[33] S. Y. Lee, Accelerator Physics, 2th ed. (World Scientific, Singapore, 2004).

[34] G. B. Arfken and H. J. Weber, Mathematical Methods for Physicists (Academic, New York, 1995).

[35] K. Gubbels, G. Meijer, and B. Friedrich, Phys. Rev. A 73, 063406 (2006).

[36] H. Dehmelt, Adv. At. Mol. Phys. 3, 53 (1967).

[37] L. D. Landau and E. M. Lifshitz, Mechanics, 3rd ed. (Pergamon, Oxford, 1976).

[38] J. van Veldhoven, J. Küpper, H. L. Bethlem, B. Sartakov, A. J. van Roij, and G. Meijer, Eur. Phys. J. D 31, 337 (2004).

[39] H. L. Bethlem, F. M. H. Crompvoets, R. T. Jongma, S. Y. T. van de Meerakker, and G. Meijer, Phys. Rev. A 65, 053416 (2002).

[40] J. van Veldhoven, H. L. Bethlem, M. Schnell, and G. Meijer, 
Phys. Rev. A 73, 063408 (2006).

[41] P. Paasche, C. Angelescu, S. Ananthamurthy, D. Biswas, T. Valenzuela, and G. Werth, Eur. Phys. J. D 22, 183 (2003).

[42] W. Petrich, M. H. Anderson, J. R. Ensher, and E. A. Cornell, Phys. Rev. Lett. 74, 3352 (1995).

[43] N. E. Shafer-Ray, K. A. Milton, B. R. Furneaux, E. R. I. Abraham, and G. R. Kalbfleisch, Phys. Rev. A 67, 045401 (2003).

[44] G. Xu, Ph.D. thesis, The University of Texas at Austin, 2001.

[45] R. Grimm, M. Weidemüller, and Y. Ovchinnikov, Adv. At., Mol., Opt. Phys. 42, 95 (1999).

[46] D. DeMille, D. R. Glenn, and J. Petricka, Eur. Phys. J. D 31, 375 (2004).

[47] The three geometries presented in this section for trapping neutral atoms and molecules using time-varying electric fields all have their magnetic counterpart. A cylindrically symmetric magnetic ac trap was proposed for spin-polarized atomic hydrogen atoms by Lovelace et al. [21], and later demonstrated for cesium atoms by Cornell et al. [22].

[48] Alternatively, one may apply a positive voltage to two neigh- bouring electrodes while the other two electrodes are kept at a negative voltage [8].

[49] When $\zeta$ and $\xi$ are chosen such that $E=0$ at the center of the trap, a hexapolar field results. An attempt to calculate $\vec{\nabla} \cdot \vec{F}$ by using Eq. (2) fails due to the $1 / E^{3}$ term. However, as the Stark effect of any molecule is quadratic in weak fields, we should use Eq. (3) instead. From Eq. (3) $\vec{\nabla} \cdot \vec{F}=0$ at the center of the trap. As both $k_{x}, k_{y}$ and $k_{z}$ are equal to zero the trap volume is infinitely small.

[50] Again, one may also apply a positive voltage to two neighbouring electrodes while the two electrodes in the same plane are kept at a negative voltage and the remaining two electrodes are at ground.

[51] Even terms will cause the force to be nonzero at the center of the trap. Peik [28] considered a small $n=2$ term to compensate the gravitational force on the atoms. In the case of polar molecules, the trap depth is so large that gravity can be neglected. 